The Astrophysical Journal Supplement Series, 173:457-470, 2007 December

(C) 2007. The American Astronomical Society. All rights reserved. Printed in U.S.A.

\title{
THE YOUNG AND THE DUSTLESS: INTERPRETING RADIO OBSERVATIONS OF ULTRAVIOLET-LUMINOUS GALAXIES
}

\author{
Antara R. Basu-Zych, ${ }^{1}$ David Schiminovich, ${ }^{1}$ Benjamin D. Johnson, ${ }^{1}$ Charles Hoopes, ${ }^{2}$ Roderik Overzier, ${ }^{3}$ \\ Marie A. Treyer, ${ }^{4,5}$ Timothy M. Heckman, ${ }^{3}$ Tom A. Barlow, ${ }^{4}$ Luciana Bianchi, ${ }^{6}$ Tim Conrow, ${ }^{4}$ \\ José Donas, ${ }^{5}$ Karl G. Forster, ${ }^{4}$ Peter G. Friedman, ${ }^{4}$ Young-Wook Lee, ${ }^{7}$ Barry F. Madore, ${ }^{8}$ \\ D. Christopher Martin, ${ }^{4}$ Bruno Milliard, ${ }^{5}$ Patrick Morrissey, ${ }^{4}$ Susan G. Neff, ${ }^{9}$ \\ R. Michael Rich, ${ }^{10}$ Samir Salim, ${ }^{3}$ Mark Seibert, ${ }^{8}$ Todd A. Small, ${ }^{4}$ \\ Alex S. Szalay, ${ }^{2}$ Ted K. Wyder, ${ }^{4}$ and Sukyoung Yi ${ }^{6}$ \\ Received 2007 February 7; accepted 2007 June 18
}

\begin{abstract}
Ultraviolet-luminous galaxies (UVLGs) have been identified as intensely star-forming nearby galaxies. A subset of these, the supercompact UVLGs, are believed to be local analogs of high-redshift Lyman break galaxies. Here we investigate the radio continuum properties of this important population for the first time. We have observed 42 supercompact UVLGs with the VLA, all of which have extensive coverage in the UV/optical by GALEX and SDSS. Our analysis includes comparison samples of multiwavelength data from the Spitzer First Look Survey and from the SDSS-GALEX matched catalogs. In addition we have Spitzer MIPS data for 24 of our galaxies and find that they fall on the radio-FIR correlation of normal star-forming galaxies. We find that our galaxies have lower radio to UV ratios and lower Balmer decrements than other local galaxies with similar (high) star formation rates. Optical spectra show they have lower $D_{n}(4000)$ and $\mathrm{H} \delta_{A}$ indices, higher $\mathrm{H} \beta$ emission-line equivalent widths, and higher $[\mathrm{O} \mathrm{III}] 5007 / \mathrm{H} \beta$ emission-line ratios than normal star-forming galaxies. Comparing these results to galaxy spectral evolution models we conclude that supercompact UVLGs are distinguished from normal star-forming galaxies firstly by their high specific star formation rates. Moreover, compared to other types of galaxies with similar star formation rates, they have significantly less dust attenuation. In both regards they are similar to Lyman break galaxies. This suggests that the process that causes star formation in the supercompact UVLGs differs from other local star-forming galaxies, but may be similar to Lyman break galaxies.
\end{abstract}

Subject headings: galaxies: starburst — radio continuum: galaxies — ultraviolet: galaxies

\section{INTRODUCTION}

GALEX has uncovered a local sample of intensely star-forming galaxies. These galaxies, referred to by Heckman et al. (2005) as ultraviolet-luminous galaxies (UVLGs), have far-ultraviolet $\left(\lambda F_{\lambda}\right.$ at $1530 \AA$ ) luminosities greater than $2 \times 10^{10} L_{\odot}$. The star formation rates (SFRs) for UVLGs range between 5 and $50 M_{\odot} \mathrm{yr}^{-1}$ (Heckman et al. 2005), which is $\approx 5-50$ times the SFR for the Milky Way. The most compact, highest surface brightness UVLGs - the supercompact UVLGs - have properties similar to those of Lyman break galaxies (LBGs) at $z>3$ (Heckman et al. 2005). Hoopes et al. (2006) has expanded the supercompact UVLG sample to confirm these findings and further determined

\footnotetext{
1 Department of Astronomy, Columbia University, 550 West 120th Street, New York, NY 10027; antara@astro.columbia.edu.

2 Department of Physics and Astronomy, Johns Hopkins University, Homewood Campus, Baltimore, MD 21218.

3 NOAO, Tuscon, Arizona.

4 California Institute of Technology, MC 405-47, 1200 East California Boulevard, Pasadena, CA 91125.

5 Laboratoire d'Astrophysique de Marseille, BP8, Traverse du Siphon, F-13376 Marseille, France.

${ }^{6}$ Center for Astrophysical Sciences, Johns Hopkins University, 3400 North Charles Street, Baltimore, MD 21218.

7 Center for Space Astrophysics, Yonsei University, Seoul 120-749, Korea.

8 Observatories of the Carnegie Institution of Washington, 813 Santa Barbara Street, Pasadena, CA 91101.

9 Laboratory for Astronomy and Solar Physics, NASA Goddard Space Flight Center, Greenbelt, MD 20771.

10 Department of Physics and Astronomy, University of California, Los Angeles, CA 90095.
}

that supercompact UVLGs also have similar metallicity to LBGs, factors of 2 or 3 less than normal galaxies of the same mass. LBGs are named for the technique used to select galaxies at high redshift based on the attenuation of their rest-frame UV continuum shortward of $\operatorname{Ly} \alpha$. These were the first high-redshift systems discovered by deep optical surveys (e.g., Hubble Deep Field, Hubble Ultra Deep Field), and comprise the most UV-luminous galaxies (Steidel et al. 1995) in the early universe.

LBGs are very biased at high redshifts (Adelberger et al. 2005), suggesting that they may be protogalaxies. Yet, since the exact start of the star formation epoch remains unknown, LBGs might rather describe a later stage in hierarchical structure formation (Steidel et al. 1999). Protogalaxies, undergoing a first intense bout of star formation, ionize surrounding hydrogen gas and emit strongly in Ly $\alpha$ before collapsing farther into fully formed galaxies, one possible explanation for the Ly $\alpha$ blobs found near LBGs at $z \sim 3.1$ (Steidel et al. 2000; Dijkstra et al. 2006; Furlanetto et al. 2005). Mori \& Umemura (2006) have conducted high-resolution hydrodynamic simulations that follow the chemical evolution of primordial galaxies, finding that LBGs resemble infant versions of elliptical and bulge systems in the local universe. At later stages of galaxy evolution, individual protogalaxies are believed to merge and form larger galaxies. Therefore, a better understanding of LBGs is essential for describing galaxy formation and evolution. Since observations at high redshifts (LBGs are found at $z \gtrsim 3$ ) suffer from lower signal-to-noise ratios and surface brightness dimming, the study of local supercompact UVLGs offers an excellent alternative for studying the physics of star formation in these distant protogalaxies. Lensed LBGs (Smail et al. 2007) can 
provide complementary approaches to studying the details of LBGs at high redshift.

Separate from the Lyman break technique, several other techniques have been used to identify intensely star-forming galaxies. As local analogs of LBGs, two classes of galaxies appear to be promising candidates based on their morphologies (Lowenthal et al. 2005). Luminous blue compact galaxies, selected by high surface brightness in the I band (Phillips et al. 1997), are intermediateredshift, blue, compact galaxies, with luminosities $\sim L^{*}$ (Garland et al. 2004, 2005; Guzmán et al. 2003); H in galaxies (or blue compact dwarfs), defined by Gil de Paz \& Madore (2005) by high surface brightness, blue colors and low stellar mass, are metal-poor starbursts with an underlying older stellar population. Significant multiwavelength work has been done to study these local, starforming galaxies in more detail (Guzmán 2005; Perez-Gonzalez et al. 2003; Rosenberg et al. 2006). While some of these samples may overlap with the UVLG sample, the supercompact UVLGs serve uniquely to compare with LBGs because of similar selection methods - both supercompact UVLGs and LBGs were selected based on UV star formation properties. Although the UV luminosities have not been studied for luminous blue compact galaxies yet, $\mathrm{H}$ II galaxies are 2 orders of magnitude less luminous in the UV (Hoopes et al. 2006), and therefore have much lower SFRs.

Analyzing star formation in galaxies provides essential clues for understanding their structure and evolution. While many star formation indicators exist, each is sensitive to a different subset of the star-forming population; the presence of dust, the metallicity, the age, or the shape of the star formation history might lead to variations in these separate diagnostics. Short-lived and massive $\mathrm{O}$ and $\mathrm{B}$ stars predominantly produce $\mathrm{UV}$ radiation. This radiation may be reprocessed by dust surrounding young $\mathrm{O}$ and $\mathrm{B}$ stars and emitted as infrared radiation. Both UV and infrared (IR) radiation trace stars formed within 10-100 million years. Another excellent tracer of star formation is the $\mathrm{H} \alpha$ produced in the $\mathrm{H}$ II regions around young $\mathrm{O}$ stars. Sensitive to only the most massive type of stars, the $\mathrm{H} \alpha$ lasts for only $\sim 5$ million years. As these massive stars die in supernovae, they produce high-energy cosmic rays. The $1.4 \mathrm{GHz}$ continuum radiation is dominated by synchrotron radiation produced by these charged particles accelerated in galactic magnetic fields. Studies by Kennicutt (1998) and Schmitt et al. (2006) have compared and correlated these various wavelength regimes, while Bell (2003), Hopkins et al. (2003), and Sullivan et al. (2001) include the discussion of timescales and star formation history on star formation indicators.

In this paper, we explore the relationship between the $1.4 \mathrm{GHz}$ radio emission and the far-ultraviolet (FUV) emission for supercompact UVLGs compared to typical galaxies, and incorporate optical spectral parameters and stellar population synthesis models to interpret our observations. Where available, our analysis is supplemented by $70 \mu \mathrm{m}$ data. Particularly, we focus on disentangling two effects using our multiwavelength observations: dust attenuation and star formation history. The radio correlates with the total SFR, while the UV measures the attenuated SFR (Bell 2003); therefore, the ratio of $1.4 \mathrm{GHz}$ SFR to FUV SFR is sensitive to the amount of attenuation. In addition, the radio signature of star formation appears on a delayed timescale $\left(\sim 3 \times 10^{6}\right.$ to $3 \times 10^{7} \mathrm{yr}$ after a single, instantaneous burst) compared to the FUV (within $3 \times 10^{6} \mathrm{yr}$ ); therefore, the offset between UV SFR and radio SFR is also affected by recent star formation history. We use various spectral measures to help separate the effect of dust attenuation from those of star formation history on the radio SFR to UV SFR ratio. We compare the radio luminosities and other properties of supercompact UVLGs with those of LBGs at higher

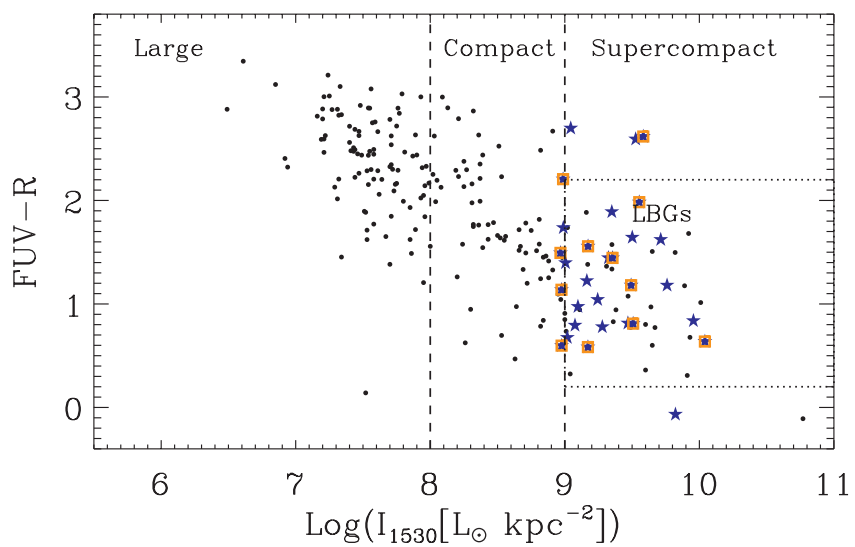

FIG. 1.-Blue stars designate the supercompact UVLGs studied in this paper. The black points come from Hoopes et al. (2006) and show how these supercompact UVLGs compare with other UVLGs in FUV surface brightness and color. We mark the VLA nondetected supercompact UVLGs with orange squares. The dashed vertical lines separate the large from the compact and supercompact UVLGs; the dotted box bounds the color and surface brightness values that describe LBGs.

redshifts (Reddy \& Steidel 2004). In $\S 2$ we introduce our various samples and describe our data analysis. We discuss our interpretation and include models in $\S 3$. Finally, we state our conclusions in $\S 4$. We adopt the following cosmology for all of our calculations: $H_{0}=70 \mathrm{~km} \mathrm{~s}^{-1} \mathrm{Mpc}^{-1}, \Omega_{m}=0.30$, and $\Lambda_{0}=0.70$.

\section{DATA AND ANALYSIS}

In this paper, we analyze how our sample of 42 supercompact UVLGs at $0.1<z<0.3$ compares with other star-forming galaxies. We employ an additional three samples that comprise local, ordinary galaxies for the same redshift range. In creating our samples of comparison galaxies, we draw from the same family of data as the supercompact UVLGs (i.e., UV-GALEX, opticalSDSS) to offer the most complementary comparisons with the least introduction of systematic errors that might arise from combining disparate data sets. The comparison samples are believed to include a heterogeneous mix of local, star-forming galaxies. In the following sections, we describe each data set separately in detail, and then compare the properties of the entire ensemble of galaxies.

\subsection{Supercompact UVLGS}

UVLGs (galaxies with $L_{\mathrm{FUV}}>10^{10.3} L_{\odot}$ ) were selected from GALEX (either MIS [Medium Imaging Survey] or AIS [All Sky Survey]; Morrissey 2007) and Sloan Digital Sky Survey (SDSS) DR2 cross matched data. Heckman et al. (2005) and Hoopes et al. (2006) separate UVLGs into three categories: supercom$\operatorname{pact}\left(I_{\mathrm{FUV}} \geq 10^{9} L_{\odot} \mathrm{kpc}^{-2}\right)$, compact $\left(10^{8} L_{\odot} \mathrm{kpc}^{-2} \leq I_{\mathrm{FUV}}<\right.$ $\left.10^{9} L_{\odot} \mathrm{kpc}^{-2}\right)$ and large $\left(I_{\mathrm{FUV}}<10^{8} L_{\odot} \mathrm{kpc}^{-2}\right)$, based on their effective radii in SDSS. Supercompact UVLGs show the most similarity with LBGs and are most intensely star forming. Here we discuss 42 supercompact UVLGs observed with the Very Large Array $\left(V_{L A}{ }^{11}\right)$. Figure 1 shows our sample of supercompact UVLGs in comparison with other UVLGs from Hoopes et al. (2006).

Our $1.4 \mathrm{GHz}$ continuum observations at the VLA were divided into two programs - one in spectral line mode (with 8 channels/ IF, each with $3.125 \mathrm{MHz}$ bandwidth), the other in continuum

11 The National Radio Astronomy Observatory is a facility of the National Science Foundation operated under cooperative agreement by Associated Universities, Inc. 

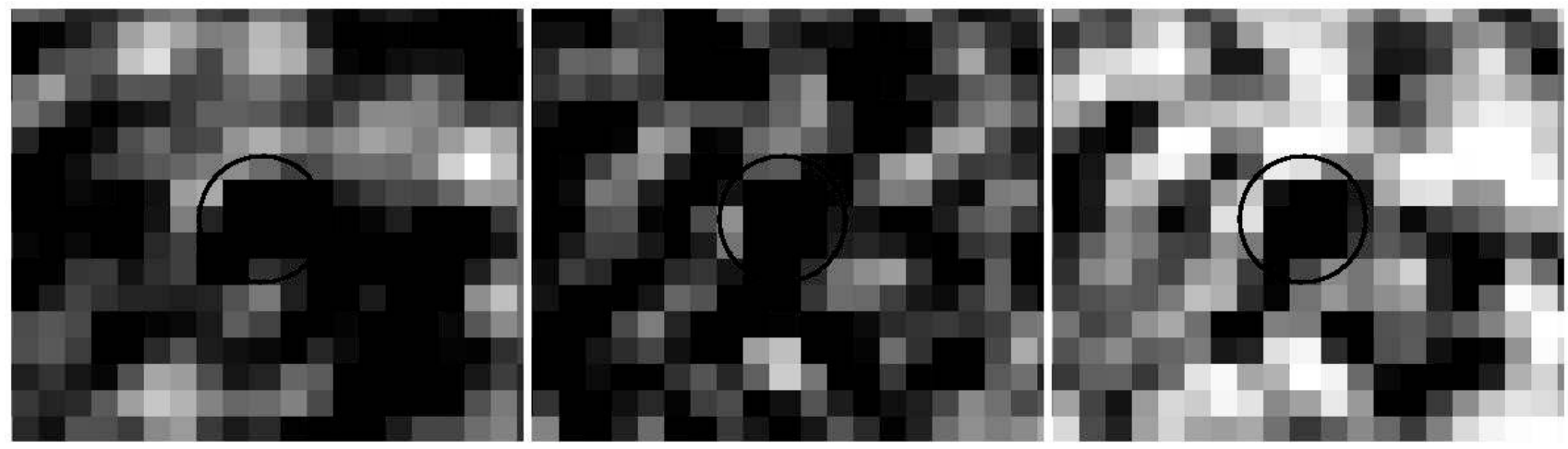

FIG. 2.-Stack 1, stack 2, and stack_all (left, middle, and right, respectively). The black circle denotes $5^{\prime \prime}$. These detections measure 141 , 157 , and $150 \mu$ Jy for stack 1 , stack 2, and stack_all, respectively, implying radio SFRs of $\sim 5.8,4.7$, and $5.2 M_{\odot} \mathrm{yr}^{-1}$.

mode. In this second program, we took our data in continuum mode with $50 \mathrm{MHz}$ bandwidth to increase our sensitivity by $\sqrt{2}$.

For our first program, $\sim 1 \mathrm{hr}$ B-array observations were taken at $20 \mathrm{~cm}$ in spectral-line mode in two IFs, centered at 1.465 and $1.385 \mathrm{GHz}$, with $25 \mathrm{MHz}$ bandwidth. We observed one of our flux calibrators $(1331+305$ [3C 286], 0542+498 [3C 147], $0137+331$ [3C 48]) for 10 minutes at the start and end of each of our three observing runs on 2005 April 11, April 13, and April 22. For each source, we applied bandpass, amplitude, and phase calibrations using standard Astronomical Image Processing System (AIPS) tasks. We cleaned a giant field of 4096 pixels $\times$ 4096 pixels $\left(2.3 \mathrm{deg}^{2}\right)$ in 1000 iterations, to find the brightest sources whose sidelobes might have contaminated the primary beam. Then we prepared field boxes from the clean components in the previous step, and 3D cleaned $\sim 0.5 \mathrm{deg}^{2}$ images down to $250 \mu \mathrm{Jy}$ (near the theoretical $5 \sigma$ ). The final images have $48 \mu \mathrm{Jy}<\mathrm{rms}<78 \mu \mathrm{Jy}$.

The analysis of the second program data was identical (with the exception of bandpass calibration) to the first run. Although our sensitivity was better in general, some fields suffered greatly from noise - without bandpass calibration, sufficient cleaning of nearby bright sources posed a challenge. Therefore, our rms spans a large range: $39 \mu \mathrm{Jy}<\mathrm{rms}<150 \mu \mathrm{Jy}$.

In the first run, 28 supercompact UVLGs were observed and 15 were detected. The second run included 15 supercompact UVLGs (eight detections and seven nondetections) from the first run, providing a consistency check between the two runs. This second run added eight new detections, bringing the final tally to 23 detected supercompact UVLGs, and $5 \sigma$ upper limits for the remaining 19. We note that the nondetections (shown by orange boxes) do not occupy any special location in Figure 1.

We stacked the nondetections from each run separately (called stack 1, with 12 galaxies, and stack 2, with nine galaxies), and combined the nondetections from both of the runs (stack_all). Some nondetections were omitted in the stacking process if the field contained nearby bright sources or excessive noise that might have contaminated the stacked image. We obtain detections of $141 \pm 21 \mu \mathrm{Jy}$ (stack 1), $157 \pm 18 \mu \mathrm{Jy}($ stack 2$)$, and $149 \pm 15 \mu \mathrm{Jy}$ (stack_all). The stacked images are shown in Figure 2. Errors were derived from the bootstrapping method. The bootstrapping technique (Efron 1982) tests how individual entries affect the stacked average. By generating a random set from the original list, we effectively duplicated some of the values while eliminating others ( $\sim 37 \%$ of the original entries got replaced by duplicate entries). We calculated a new average. This process was repeated
$N \log (N)$ times, where $N$ is the number of samples - in this case the number of images stacked to produce one stacked image. The standard deviation of these simulated data corresponds to the error in our stacked result.

The radio emission was $k$-corrected by $(1+z)^{\alpha}$ with $\alpha=0.8$, since we assumed a radio spectrum of the form $S_{\nu} \propto \nu^{-0.8}$. We consistently use the integrated radio intensities throughout this paper, unless explicitly stated otherwise. We derived the FUV luminosities from magnitudes given in the GALEX catalog. For cases where GALEX observed one supercompact UVLG on multiple visits, we computed an exposure-weighted average magnitude. The FUV magnitude was Galactic extinction corrected by $8.24 E(B-V)$, determined using Wyder (2007), and $k$-corrected applying the kcorrect_4.1.4 IDL routine (Blanton et al. 2003; using the optical $u^{\prime} g^{\prime} r^{\prime} i^{\prime} z^{\prime}$ bands from SDSS and FUV, NUV from GALEX for spectral fitting). Table 1 summarizes the observations for the supercompact UVLGs. We include $70 \mu \mathrm{m}$ Spitzer data for comparison. We used the $70 \mu \mathrm{m}$ mosaic images created by the Spitzer Science Center pipeline and measured the fluxes of point sources at the expected locations of the supercompact UVLGs using $30^{\prime \prime}$ circular apertures in IRAF. The aperture corrections given on the Spitzer Web site were applied.

\subsection{Comparison Sample 1: GALEX MIS, SDSS DR2, and VLA}

As described above, the VLA fields from our first program were centered on supercompact UVLGs. The spectral line mode in the first program allowed us to obtain $1.4 \mathrm{GHz}$ data for serendipitous galaxies in the same fields, since the bandpass calibration made possible the proper cleaning of wider images. After applying the AIPS data reduction tasks, we found a total of 24 other galaxies in these fields that existed in both the SDSS DR2 and GALEX GR1 MIS data (separate from the 15 supercompact UVLGs), which we believe to represent a sample of star-forming galaxies. We refer to these galaxies as our MIS/DR2/VLA set. The stellar mass was derived for these galaxies according to the prescription in Brinchmann et al. (2004). The UV, radio, and optical properties were determined in the same way as for the supercompact UVLGs.

Since galaxies containing active galactic nuclei (AGNs)strongly emitting in UV and radio through processes unrelated to star formation - could contaminate our assessment of star-forming properties, we eliminated AGNs by using the $D_{n}(4000)$ and the radio luminosity per stellar mass as described by Best et al. (2005) (Fig. 3). As discussed later in this paper, $D_{n}(4000)$ is a common measure of stellar age. Star-forming galaxies with higher $D_{n}(4000)$ values contain older stars and lower $L_{\text {radio }} / M_{*}$, since $L_{\text {radio }} / M_{*}$ 
TABLE 1

SUPERCOMPACT UVLGs

\begin{tabular}{|c|c|c|c|c|c|c|c|}
\hline SDSS ID & $z$ & $\begin{array}{l}S_{70 \mu \mathrm{m}} \\
(\mathrm{mJy})\end{array}$ & $\begin{array}{c}S_{1.4 \mathrm{GHz}}{ }^{\mathrm{a}} \\
(\mu \mathrm{Jy})\end{array}$ & $\begin{array}{c}L_{1.4 \mathrm{GHz}^{\mathrm{b}}} \\
\left(10^{22} \mathrm{~W} \mathrm{~Hz}^{-1}\right)\end{array}$ & $\begin{array}{l}\mathrm{SFR}_{1.4 \mathrm{GHz}} \\
\left(M_{\odot} \mathrm{yr}^{-1}\right)\end{array}$ & $\begin{array}{c}L_{\mathrm{FUV}}^{\mathrm{b}} \\
\left(10^{22} \mathrm{~W} \mathrm{~Hz}^{-1}\right)\end{array}$ & $\begin{array}{c}\mathrm{SFR}_{\mathrm{FUV}} \\
\left(M_{\odot} \mathrm{yr}^{-1}\right)\end{array}$ \\
\hline SDSS J205000.00+003124.7 ……................ & 0.164 & $\ldots$ & $<324$ & $<2.3$ & $<8.5$ & $0.15 \pm 0.07$ & $1.4 \pm 0.6$ \\
\hline SDSS J232539.22+004507.2 ….................. & 0.277 & $<17$ & $338 \pm 54$ & $7.7 \pm 1.2$ & $28.2 \pm 4.5$ & $0.41 \pm 0.13$ & $3.8 \pm 1.2$ \\
\hline SDSS J232624.84+134206.4 …….................. & 0.207 & $\ldots$ & $<295$ & $<3.5$ & $<12.9$ & $0.95 \pm 0.14$ & $8.8 \pm 1.3$ \\
\hline SDSS J001009.97-004603.6......................... & 0.243 & $<12$ & $<215$ & $<3.7$ & $<13.4$ & $0.37 \pm 0.13$ & $3.5 \pm 1.2$ \\
\hline SDSS J021348.53+125951.4 ………........... & 0.219 & $\ldots$ & $896 \pm 68$ & $12.1 \pm 0.9$ & $44.3 \pm 3.4$ & $0.72 \pm 0.22$ & $6.7 \pm 2.1$ \\
\hline SDSS J032845.99+011150.8 ….................... & 0.142 & $<11$ & $<240$ & $<1.3$ & $<4.6$ & $0.34 \pm 0.12$ & $3.2 \pm 1.1$ \\
\hline SDSS J035733.99-053719.7 ........................ & 0.204 & $49 \pm 6$ & $444 \pm 85$ & $5.1 \pm 1.0$ & $18.7 \pm 3.6$ & $0.43 \pm 0.16$ & $4.0 \pm 1.5$ \\
\hline SDSS J081523.39+500414.6 …….............. & 0.164 & $\ldots$ & $672 \pm 63$ & $4.9 \pm 0.5$ & $17.7 \pm 1.7$ & $0.54 \pm 0.07$ & $5.0 \pm 0.7$ \\
\hline SDSS J101211.18+632503.6............................ & 0.246 & $<16$ & $<230$ & $<4.0$ & $<14.7$ & $0.28 \pm 0.10$ & $2.6 \pm 0.9$ \\
\hline SDSS J101741.02+510438.4 .......................... & 0.213 & $\ldots$ & $<234$ & $<3.0$ & $<10.8$ & $0.49 \pm 0.07$ & $4.5 \pm 0.7$ \\
\hline SDSS J223429.58-092452.9 …................... & 0.246 & $\ldots$ & $<373$ & $<6.5$ & $<23.9$ & $0.17 \pm 0.08$ & $1.6 \pm 0.8$ \\
\hline SDSS J230703.76+011311.1 …...................... & 0.126 & $\ldots$ & $664 \pm 76$ & $2.7 \pm 0.3$ & $9.8 \pm 1.1$ & $0.41 \pm 0.05$ & $3.8 \pm 0.4$ \\
\hline SDSS J214500.25+011157.5 ……................. & 0.204 & $23 \pm 5$ & $341 \pm 69$ & $4.0 \pm 0.8$ & $14.5 \pm 2.9$ & $0.37 \pm 0.08$ & $3.4 \pm 0.7$ \\
\hline SDSS J231812.99-004126.1 ……................. & 0.252 & $65 \pm 4$ & $679 \pm 72$ & $12.5 \pm 1.3$ & $45.7 \pm 4.8$ & $1.03 \pm 0.12$ & $9.6 \pm 1.1$ \\
\hline SDSS J001054.85+001451.3 ……................ & 0.243 & $111 \pm 5$ & $753 \pm 74$ & $12.9 \pm 1.3$ & $47.1 \pm 4.6$ & $0.43 \pm 0.09$ & $4.0 \pm 0.8$ \\
\hline SDSS J004447.33+152911.7 ……................. & 0.227 & $\ldots$ & $462 \pm 78$ & $6.8 \pm 1.1$ & $24.8 \pm 4.2$ & $0.53 \pm 0.09$ & $4.9 \pm 0.8$ \\
\hline SDSS J005527.46-002148.7 ….................... & 0.167 & $163 \pm 6$ & $542 \pm 72$ & $4.1 \pm 0.5$ & $14.9 \pm 2.0$ & $0.45 \pm 0.08$ & $4.2 \pm 0.8$ \\
\hline SDSS J024529.55-081637.7 ……................ & 0.196 & $\ldots$ & $373 \pm 67$ & $3.9 \pm 0.7$ & $14.4 \pm 2.6$ & $0.35 \pm 0.06$ & $3.3 \pm 0.5$ \\
\hline SDSS J040208.86-050642.0 ….................... & 0.139 & $<20$ & $<225$ & $<1.1$ & $<4.1$ & $0.40 \pm 0.07$ & $3.7 \pm 0.6$ \\
\hline SDSS J092336.45+544839.2 …...................... & 0.222 & $45 \pm 4$ & $<276$ & $<3.9$ & $<14.1$ & $0.49 \pm 0.08$ & $4.6 \pm 0.7$ \\
\hline SDSS J210358.74-072802.4 ........................ & 0.137 & $421 \pm 6$ & $3840 \pm 48$ & $18.6 \pm 0.2$ & $68.1 \pm 0.9$ & $0.54 \pm 0.24$ & $5.0 \pm 2.2$ \\
\hline SDSS J004054.32+153409.6....................... & 0.283 & $25 \pm 5$ & $<220$ & $<5.3$ & $<19.3$ & $0.24 \pm 0.09$ & $2.2 \pm 0.9$ \\
\hline SDSS J092159.38+450912.3 …..................... & 0.235 & $228 \pm 8$ & $1410 \pm 51$ & $22.3 \pm 0.8$ & $81.5 \pm 2.9$ & $0.96 \pm 0.17$ & $8.9 \pm 1.6$ \\
\hline SDSS J092600.40+442736.1 ….................... & 0.181 & $21 \pm 4$ & $242 \pm 42$ & $2.1 \pm 0.4$ & $7.8 \pm 1.4$ & $0.74 \pm 0.07$ & $6.8 \pm 0.6$ \\
\hline SDSS J124819.74+662142.6 ……............. & 0.260 & $\ldots$ & $<320$ & $<6.3$ & $<23.2$ & $0.54 \pm 0.12$ & $5.0 \pm 1.1$ \\
\hline Stack $1^{\mathrm{c}}$ & 0.22 & $\ldots$ & $141 \pm 21$ & $1.6 \pm 0.2$ & $5.8 \pm 1.1$ & $0.45 \pm 0.09$ & $4.2 \pm 0.8$ \\
\hline Stack $2^{\mathrm{c}}$.. & 0.22 & $\ldots$ & $157 \pm 18$ & $1.3 \pm 0.1$ & $4.7 \pm 0.9$ & $0.45 \pm 0.10$ & $4.2 \pm 1.0$ \\
\hline Stack_all ${ }^{c}$ & 0.22 & $\ldots$ & $149 \pm 15$ & $1.4 \pm 0.1$ & $5.2 \pm 0.7$ & $0.45 \pm 0.10$ & $4.2 \pm 1.0$ \\
\hline
\end{tabular}

\footnotetext{
${ }^{\text {a }} S_{1.4 \mathrm{GHz}}$ gives the measured (not $k$-corrected) value.

b Luminosities were calculated using the following cosmology: $H_{0}=70 \mathrm{~km} \mathrm{~s}^{-1} \mathrm{Mpc}^{-1}, \Omega_{M}=0.3, \Omega_{\nu}=0.7$. We compute exposure weighted averages from the FUV magnitudes.

${ }^{c}$ Stack 1 includes all nondetected galaxies from our first VLA observation. Stack 2 includes the nondetected galaxies from the second VLA observation. Finally, stack_all includes the galaxies from both stack 1 and stack 2 . Details about the stacking process are discussed in the text. The redshift, radio flux density, radio and FUV luminosities, and SFRs are all averaged values.
}

gauges the present to past SFR. Therefore radio emission for galaxies above the dotted line in Figure 3, with high $L_{\text {radio }} / M_{*}$, must be dominated by AGN activity rather than star formation. We also note that by this method supercompact UVLGs (blue stars) do not show any evidence of AGN activity, consistent with the selection criterion that Hoopes et al. (2006) employed.

\subsection{Comparison Sample 2: First Look Survey Galaxies}

The Spitzer First Look Survey (FLS) covered $5 \mathrm{deg}^{2}$ centered at R.A. $=17^{\mathrm{h}} 18^{\mathrm{m}}$, decl. $=+59^{\circ} 30^{\prime}(\mathrm{J} 2000.0)$. This region has excellent wavelength coverage, including observations in the IR by Spitzer Space Telescope, radio by the VLA, optical by SDSS, and ultraviolet by GALEX. In this study, we restricted our analysis to the SDSS, radio, and ultraviolet data. We considered only the sources that have FUV and radio detections. We applied the process described above to remove galaxies dominated by AGN activity, $\sim 30 \%$ of our sample. After these steps, our sample contains FUV and radio luminosities for 189 galaxies.

The GALEX observations were $\sim 20$ ks and achieved FarUltraviolet (FUV) magnitudes $\leq 24.7$. VLA observed the FLS galaxies in B array at $20 \mathrm{~cm}$, achieving sensitivities $S>115 \mu \mathrm{Jy}$ 


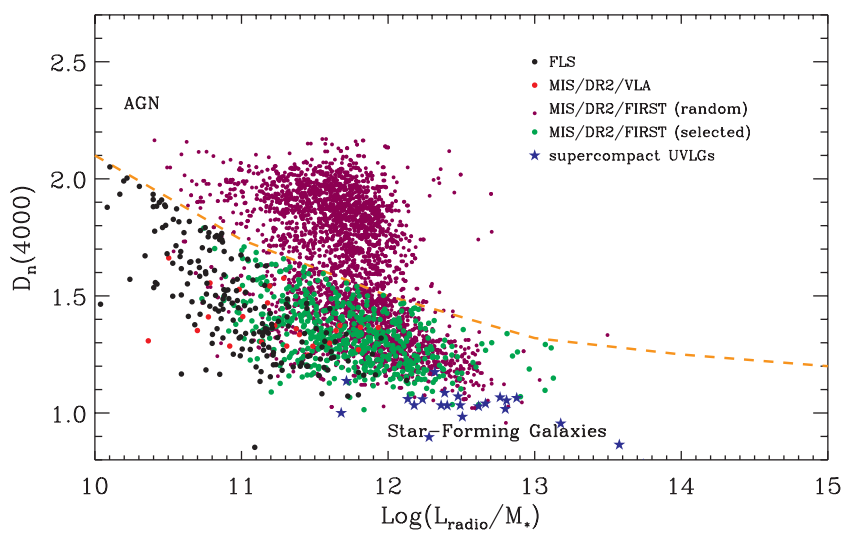

FIG. 3.-Using Best et al. (2005) to apply an AGN cut: the galaxies above the orange line are designated AGNs and excluded from our analysis, while the galaxies below the line remain in our samples. We indicate FLS galaxies with black points and the MIS/DR2/VLA sample with red points. The smaller purple points display a random set from the full 60,000 MIS/DR2/FIRST galaxies, whereas the green points show the same data set once AGNs have been removed. Supercompact UVLGs appear as blue stars.

(see Condon et al. [2003] for full details regarding the VLA observations).

\subsection{Comparison Sample 3: GALEX MIS, SDSS DR2, and FIRST}

The matched catalogs from SDSS and the GALEX Internal Release 1.1 MIS comprise data for $\sim 45,000$ galaxies. As described for the MIS/DR2/VLA galaxies, we eliminated the AGNs, and $\sim 21,000$ galaxies remained in our sample. Faint Images of the Radio Sky at Twenty cm (FIRST) has observed most of these galaxies in brief 3 minute VLA snapshots in B array (Becker et al. $1995)$. Very few $(<3 \%)$ of the of MIS/DR2 galaxies were detected by FIRST. However, we stacked the FIRST observations to simulate deeper observations of nonradio selected galaxies (such as the supercompact UVLGs, therefore providing an important comparison).

To perform our stacking, we separated the galaxies into bins by FUV luminosity (from $17.5<\log L_{\mathrm{FUV}}<22.5$, in increments of 0.1 ), redshift (from $0.05<z<0.35$ in increments of 0.05 ), and attenuation $(0.0<\tau<1.0) .{ }^{12}$ We calculated a stacked image by taking the mean of the images for each bin, provided that the bin contained at least 30 galaxies. These stacked "detections," henceforward the MIS/DR2/FIRST galaxies, provide an average measurement of the radio luminosity for typical MIS/DR2 galaxies. In this analysis, the radio intensities are the peak intensities. We assumed that the galaxies are unresolved and the peak intensity could be approximated as the integrated intensity for the stacked galaxies. This might lead to an underestimate in the radio luminosity for the nearby galaxies; FIRST's resolution is $5^{\prime \prime}$, implying that galaxies larger than $11 \mathrm{kpc}$ at $z=0.1$ would be resolved, while galaxies at $z=0.3$ would remain unresolved at sizes as large as $38 \mathrm{kpc}$.

A small number of galaxies $(\sim 2.5 \%)$ in this MIS/DR2/FIRST sample were detected by FIRST. We included these detections in calculating the average radio intensity for the relevant redshift and $L_{\mathrm{FUV}}$ bin. However, the detected sources were excluded from the stacking process. We applied the correction prescribed in White et al. (2006) to account for the snapshot bias of unknown origin. This snapshot bias leads to an underestimate of the source flux (even

\footnotetext{
${ }^{12} \tau \equiv \ln [(\mathrm{H} \alpha / \mathrm{H} \beta)(1 / 2.88)]$ (Calzetti et al. 1994). Our use of $\tau$ for measuring attenuation is discussed further in the discussion.
}

for stacked sources) because the nonlinear cleaning process redistributes flux from sources to the background. To estimate errors in our stacked image we used the bootstrapping method described earlier. In addition, we consider the detections as a separate comparison sample, referred to as the MIS/DR2/FIRST (det) galaxies.

\subsection{Computing Radio and UV SFRs}

SFRs were derived from the FUV and radio luminosities using the relations of Hopkins et al. (2003). The FUV SFR derivation agrees with that of Kennicutt (1998) and Sullivan et al. (2001). The radio SFR relation matches the derivation given in Bell (2003), which normalizes the radio SFR to match the IR SFR for high-luminosity galaxies. We note that this conversion to the radio SFR differs from that in Condon (1992) and Sullivan et al. (2001) by a factor of 2 for $L_{1.4 \mathrm{GHz}}>6.4 \times 10^{21} \mathrm{~W} \mathrm{~Hz}^{-1}$ (see discussions in Bell [2003] and Hopkins et al. [2003]).

Hopkins et al. (2003) used the Salpeter (1955) initial mass function (IMF) $\left[\Psi(M) \sim M^{-2.35}\right]$, and a mass range of $0.1<$ $M_{\odot}<100$. In order to adopt the Kroupa (2001) IMF, we divided the SFR conversions given in Hopkins et al. (2003) by 1.5. The $\mathrm{UV}$ and radio SFRs that we used were

$$
\begin{gathered}
\operatorname{SFR}_{\mathrm{FUV}}\left(M_{\odot} \mathrm{yr}^{-1}\right)=\frac{L_{\mathrm{FUV}}}{1.07 \times 10^{21}} \\
\operatorname{SFR}_{1.4 \mathrm{GHz}}\left(M_{\odot} \mathrm{yr}^{-1}\right)= \\
\begin{cases}\frac{L_{1.4 \mathrm{GHz}}}{2.72 \times 10^{21}} & \text { if } L_{1.4 \mathrm{GHz}}>L_{c}, \\
\frac{L_{1.4 \mathrm{GHz}}}{2.72 \times 10^{21}\left[0.1+0.9\left(L_{1.4 \mathrm{GHz}} / L_{c}\right)^{0.3}\right]} & \text { if } L_{1.4 \mathrm{GHz}} \leq L_{c},\end{cases}
\end{gathered}
$$

where $L_{c}=6.4 \times 10^{21} \mathrm{~W} \mathrm{~Hz}^{-1}$, and $L_{\mathrm{FUV}}$ and $L_{1.4 \mathrm{GHz}}$ are in $\mathrm{W} \mathrm{Hz}^{-1}$. We used equations (1) and (2) to derive all the UV and radio SFRs throughout this paper. SDSS optical spectral properties have been computed and made available online. ${ }^{13}$ For our analysis in this paper, we used the optical line fluxes (described in Tremonti et al. 2004), stellar masses, $\mathrm{H} \delta$ absorption line $\left(\mathrm{H} \delta_{A}\right)$, and $4000 \AA$ break index $\left[D_{n}(4000)\right.$; detailed in Brinchmann et al. 2004] from these tables. We note that the UV SFRs and luminosities throughout this paper (for all the samples) are uncorrected for dust attenuation.

\subsection{The Ensemble}

In this paper, we focus on how supercompact UVLGs compare with other star-forming galaxies. The FLS, MIS/DR2/FIRST, and MIS/DR2/VLA data constitute our comparison samples. In Table 2 we summarize the separate data sets; Figure 4 illustrates how the physical quantities (redshift, UV and radio luminosities, $D_{n}(4000), \tau$, and $\left.\mathrm{H} \delta_{A}\right)$ in the samples are distributed. We note the following:

1. All the comparison samples agree in their distributions for redshifts and UV and radio luminosities. The median redshifts for the MIS/DR2/VLA, MIS/DR2/FIRST detected and stacked points, and FLS are: $0.07,0.08,0.08$, and 0.1 (the standard deviation for all samples is 0.05 ). The median UV luminosity (in log units) for the same aforementioned data sets are $20.8 \pm 0.5,20.6 \pm 0.6$, $20.5 \pm 0.6$, and $20.7 \pm 0.5$; similarly, the median radio luminosity are $22.1 \pm 0.6,22.5 \pm 0.5,21.7 \pm 0.6$, and $21.9 \pm 0.6$. The stacked MIS/DR2/FIRST galaxies include less luminous radio

${ }^{13}$ See http://www.mpa-garching.mpg.de/SDSS. 


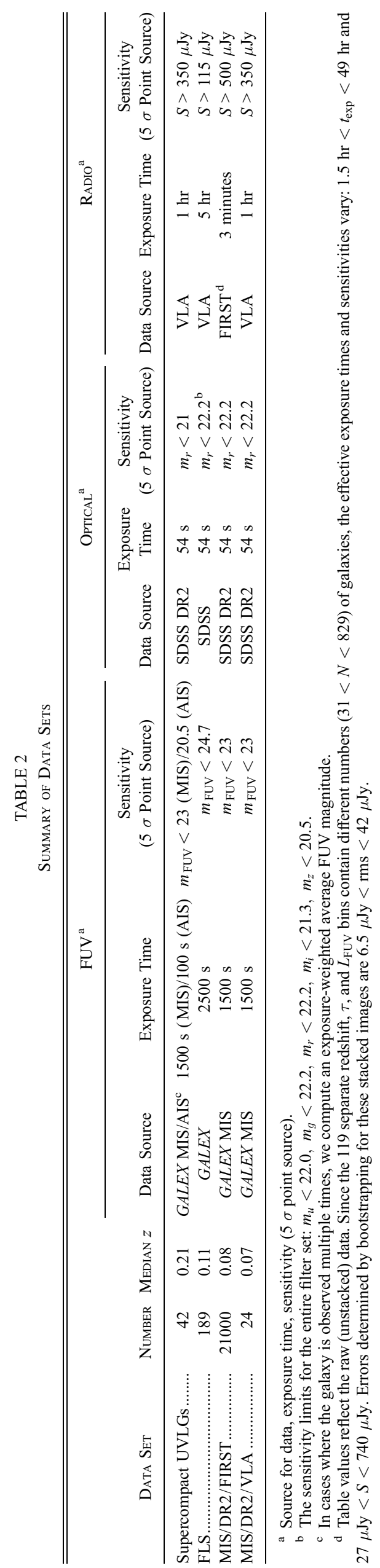



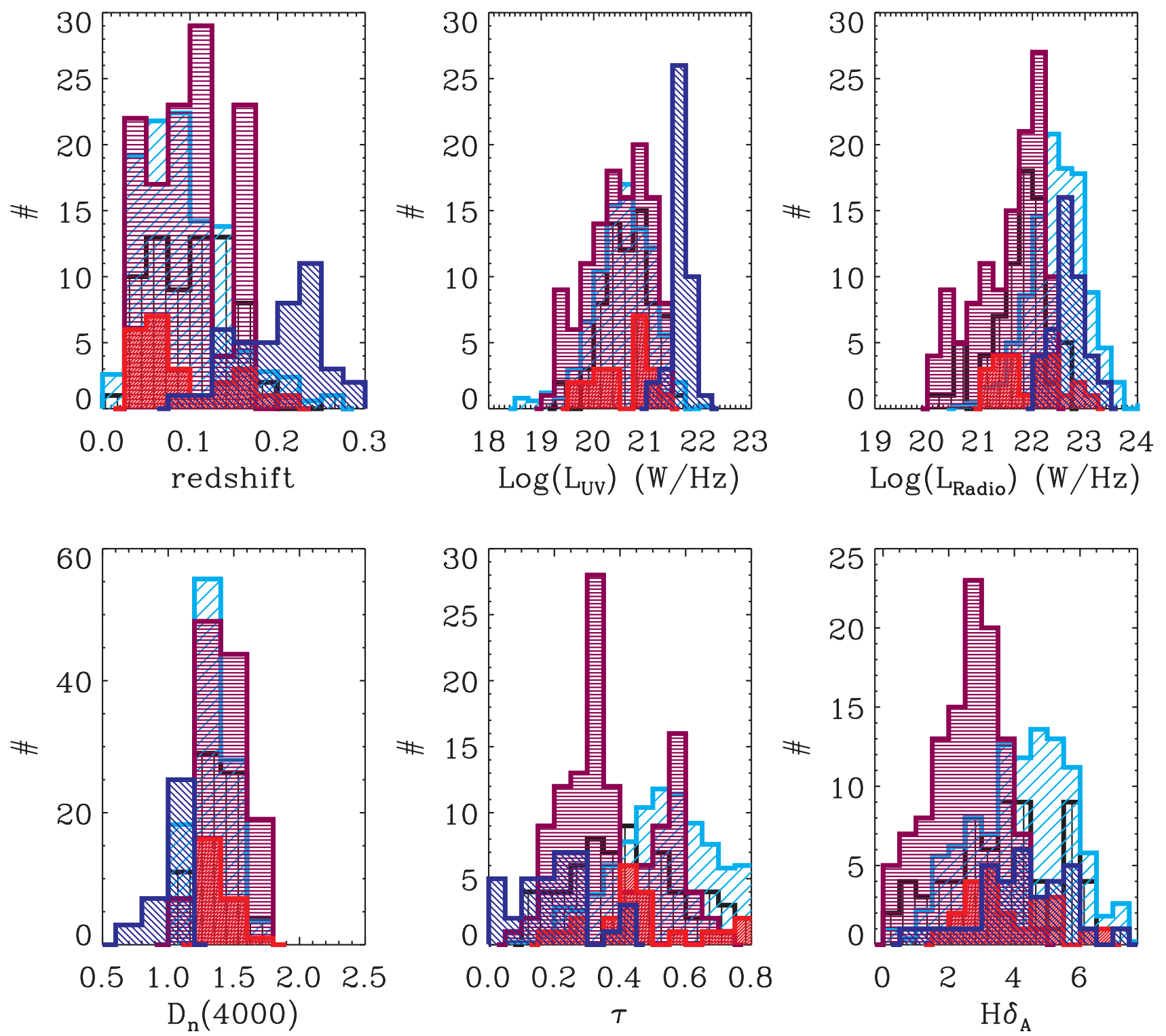

FIg. 4.-Comparison of redshifts, UV luminosities, radio luminosities, $D_{n}(4000), \tau$ (see footnote 12), and $\mathrm{H} \delta_{A}$ for the samples (we show the total number for each sample in parentheses). Blue, negatively sloped diagonal stripes describe supercompact UVLGs (42); violet horizontal stripes show the stacked MIS/DR2/FIRST sample (99); cyan, positively sloped diagonal stripes display MIS/DR2/FIRST detections (526, we scaled this distribution down by a factor of 5 in order to display all the data sets clearly); red, positively sloped diagonal stripes display MIS/DR2/VLA galaxies (27); black vertical stripes illustrate FLS galaxies (79).

and UV galaxies, since the longer effective exposure times allow for deeper observations, while MIS/DR2/VLA and MIS/DR2/FIRST (detected) galaxies correspond to more luminous radio sources.

2. Supercompact UVLGs have a redshift distribution peaking at a slightly higher redshift (their median redshift is $0.21 \pm 0.05$ ). The distributions of UV and radio luminosities are more sharply peaked and at higher values than the other data sets (median values of UV luminosity and radio luminosity are $21.6 \pm 0.2$ and $22.7 \pm 0.3$ in log units). This is not surprising, since supercompact UVLGs are restricted to those galaxies with UV luminosities greater than $2 \times 10^{10} L_{\odot}$.

3. The comparison samples, MIS/DR2/VLA, MIS/DR2/ FIRST (detected), and FLS, were selected to have detections in both the FUV and the radio. Including radio nondetections might have given a closer comparison to the supercompact UVLGs, which were not radio selected, and would probe the relations at lower radio luminosities. The stacked nondetections of the MIS/ DR2/FIRST galaxies serve this purpose.
In summary, the FLS and stacked MIS/DR2/FIRST data provide the deepest observations in both radio and UV. The FLS, MIS/DR2/VLA, and MIS/DR2/FIRST (detected) samples include radio- and UV-selected galaxies, while the supercompact UVLGs are only UV selected. In fact, the MIS/DR2/FIRST (detected) sample includes the most radio-luminous galaxies, a subset of which may be IR-luminous galaxies. We include the stacked MIS/DR2/FIRST galaxies for two reasons: to extend our sample to lower radio luminosities, and to compare the supercompact UVLGs with galaxies that were also not radio selected. Where appropriate, we will provide two versions of our plots: one excluding the stacked MIS/DR2/FIRST data to provide a less cluttered view, and the other with the full set.

\section{DISCUSSION}

Why are UVLGs so luminous in the UV? Might they be highly obscured galaxies with high SFRs, that still produce significant UV luminosity? Or do these galaxies have moderate SFRs, but 


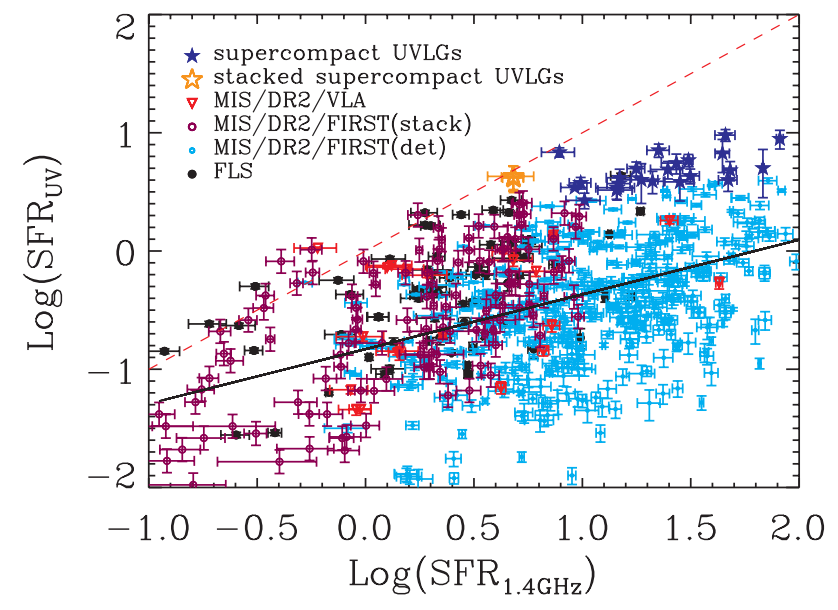

FIG. 5.-Comparison of the unattenuated SFR $\left(\mathrm{SFR}_{\text {radio }}\right)$ with the attenuated SFR $\left(\mathrm{SFR}_{\mathrm{uv}}\right)$. Supercompact UVLGs (blue stars) seem to have slightly higher UV emission (or lower radio emission) compared to the other local galaxies (First Look Survey galaxies shown by black dots; MIS/DR2/VLA galaxies are shown as inverted red triangles; stacked MIS/DR2/FIRST galaxies are displayed as violet open circles; detected MIS/DR2/FIRST galaxies are indicated by cyan points). The solid black line designates the best fit line to all the points (we display this fit in order to guide the eye, without intending to quantify any physical connection between the two axes); the dashed red line marks equivalent radio and UV SFR.

less attenuation from dust compared to other UV and opticallyselected galaxies? Comparing the radio SFR to the UV SFR (Fig. 5) shows that supercompact UVLGs have higher star formation in both diagnostics compared to the galaxies in our comparison samples. It should be noted that if we include a sample of IR luminous and ultraluminous galaxies in this figure they would likely fall in the region below the supercompact UVLGs. For all of the galaxies, including the supercompact UVLGs, the UV SFR and radio SFR measures are consistent. The dashed red line shows the line of equivalent SFR. We expect that the actual UV SFR underestimates the total SFR (here, taken to be the radio SFR) because UV suffers strongly from dust attenuation, if present. The shallower slope of the solid black line (the best-fit line to all the data points, including the supercompact UVLGs) compared to the dashed red line suggests that attenuation increases with increasing SFR (similarly, Wang \& Heckman [1996] and Martin et al. [2005] show that dust extinction increases with both far-infrared (FIR) and FIR+UV luminosities for normal late-type galaxies). All the samples of regular galaxies scatter around the fit (black solid line) reasonably well. We provide the solid black line as a visual reference for describing the trend outlined by all the galaxies in our samples but note that the fit is not physically motivated, nor intended to quantitatively relate the radio SFR to the UV SFR. Compared to this trend, the supercompact UVLGs are offset- these galaxies seem underluminous in their radio emission compared to the UV. We note, however, that the median radio luminosity for the supercompact UVLGs, $L_{1.4 \mathrm{GHz}} \sim 5.8 \times 10^{22} \mathrm{~W} \mathrm{~Hz}^{-1}$, is consistent with the value determined for LBGs (by stacking LBGs at $1.5 \geq z \geq 3.0$ from the Great Observatories Origins Deep SurveyNorth field), $L_{1.4 \mathrm{GHz}} \sim(5.90 \pm 1.66) \times 10^{22} \mathrm{~W} \mathrm{~Hz}^{-1}$ (Reddy \& Steidel 2004).

We briefly consider the IR data for supercompact UVLGs, comparing our $L_{1.4 \mathrm{GHz}}$ and $L_{60 \mu \mathrm{m}}$ values with those from Yun et al. (2001). We compute the $60 \mu \mathrm{m}$ rest frame luminosities, $L_{60 \mu \mathrm{m}}=$ $\nu_{60 \mu \mathrm{m}} F_{60 \mu \mathrm{m}}$, from the observed $70 \mu \mathrm{m}$ Spitzer data. From Figure 6, we find that the supercompact UVLGs appear to follow the radio-IR correlation. We plan to complete further analysis of the

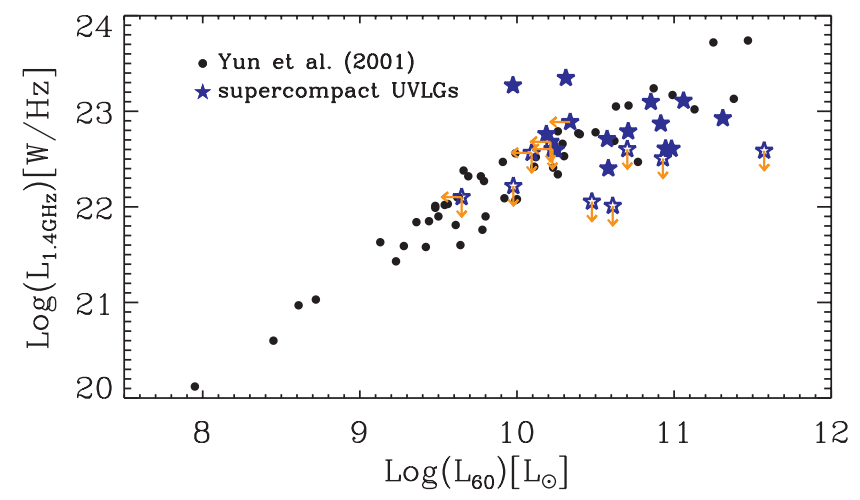

FIG. 6.-Radio vs. IR: Comparison of the $60 \mu \mathrm{m}$ with $1.4 \mathrm{GHz}$ radiation. The supercompact UVLGs follow the points from Yun et al. (2001). Radio or IR nondetections appear as open stars with orange arrows denoting $5 \sigma$ upper limits for the $60 \mu \mathrm{m}$ (arrows pointing left) and the $1.4 \mathrm{GHz}$ (arrows pointing down) data; radio detections are shown by filled stars.

other IR bands to determine the total IR luminosity and IR SFR in a future paper.

Assuming that the radio SFR measures the total SFR we can define $L_{\text {tot, rad }}=5.4 \times 10^{9} \mathrm{SFR}_{\text {rad }}$ (Martin et al. 2005; Kennicutt 1998), where $L_{\text {tot,rad }}$ is the equivalent bolometric luminosity of young, massive stars as derived from our radio luminosities. Given that the UV SFR underestimates the total star formation proportional to the amount dust attenuation and the SFR derived from the IR makes up this difference, then $\mathrm{SFR}_{\mathrm{IR}}+\mathrm{SFR}_{\mathrm{UV}}=\mathrm{SFR}_{\mathrm{rad}}$. Therefore $L_{\mathrm{IR}}+L_{\mathrm{UV}}=L_{\mathrm{tot}, \text { rad }}$. In the top panel of Figure 7, we plot $\log \left(L_{\text {tot, rad }}\right)$ versus $\log \left[\left(L_{\text {tot, rad }}-L_{\mathrm{UV}}\right) / L_{\mathrm{UV}}\right]$, similar to Wang \& Heckman (1996) and Martin et al. (2005). The solid line gives
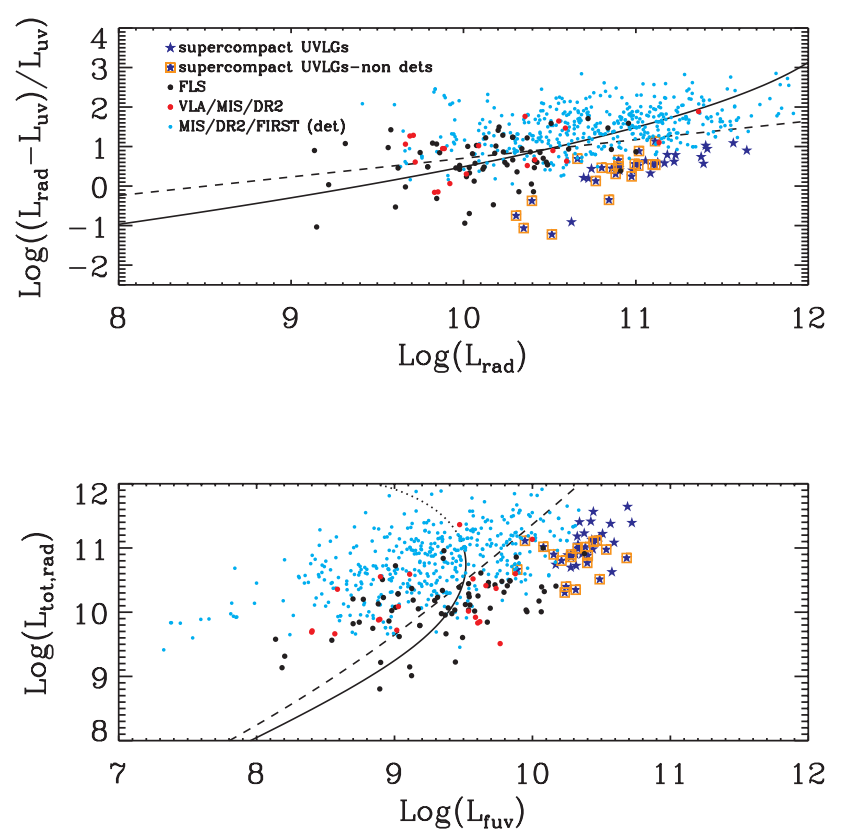

FIG. 7.- Radio luminosity vs. IR/UV luminosity (top) with fits from Martin et al. (2005; solid lines) and Wang \& Heckman (1996; dashed lines). UV luminosity vs. radio luminosity (bottom). The lines show the radio luminosity derived from Martin et al. (2005; solid lines) and Wang \& Heckman (1996; dashed lines). The FLS and VLA/MIS/DR2 galaxies have scatter both above and below the fits, while the supercompact UVLGs all have radio luminosities that fall below the relation. 

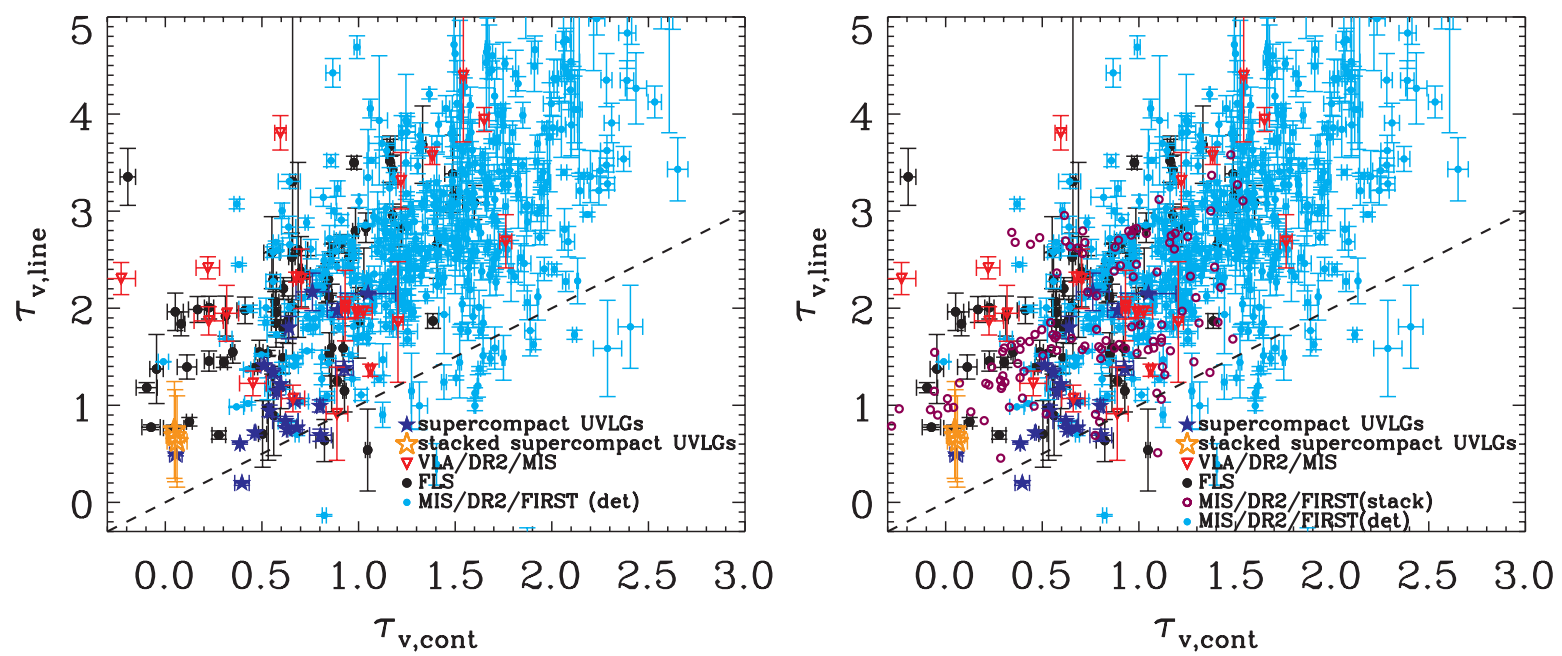

FIG. 8. - Attenuation in the $V$ magnitude of the $\mathrm{H} \alpha / \mathrm{H} \beta$ lines $\left(\tau_{V \text {,line }}\right)$ vs. the continuum $\left(\tau_{V \text {,cont }}\right)$, measured from radio-to-UV SFR ratio (See eqs. [9] and [10]). The samples are the same as in Fig. 4. The left panels exclude the stacked MIS/DR2/FIRST galaxies to visually simplify the plot, while the panels on the right display the full set. The dashed line shows where $\tau_{V \text {,line }}=\tau_{V \text {, cont }}$.

the fit from Martin et al. (2005) and the dashed line shows the fit from Wang \& Heckman (1996). We see that the samples of normal galaxies have scatter both above and below the fits, but the supercompact UVLGs fall below the relations. In the bottom panel, we plot radio versus UV luminosity, and the lines show the radio luminosity derived from the UV luminosity and the fit from Martin et al. (2005; solid line) and from Wang \& Heckman (1996; dashed line). Here, we can clearly see that all the supercompact UVLGs in our sample have radio luminosities lower than expected based on their UV-derived SFR.

We explore a couple of scenarios which might explain the lower radio to UV emission: first, supercompact UVLGs could be less attenuated; secondly, they could be dominated by younger, lessevolved stellar populations compared to other galaxies.

Examining the first option, we determine the dust content of the samples using two independent techniques. The first method uses the ratio of radio to UV radiation from these galaxies. As discussed above, the UV luminosity gives us a measure of the attenuated SFR, while the radio emission can be used to measure the unattenuated SFR. The other technique uses the ratio of line fluxes from $\mathrm{H} \alpha$ and $\mathrm{H} \beta: \tau \equiv \ln [(\mathrm{H} \alpha / \mathrm{H} \beta)(1 / 2.88)]$. Both measure the amount of hydrogen in the system, but $\mathrm{H} \beta$ is more sensitive to dust. The natural difference between the line strengths is accounted for by the factor 2.88. Larger values of $\tau$ indicate greater attenuation. Note that the Balmer decrement, or $\tau$, may be a upper limit to the actual amount of attenuation, since emission lines tend to show more attenuation than continuum (Charlot $\&$ Fall 2000).

Figure 8 compares the line with the continuum attenuation. To better compare the two attenuation values, we convert both to $\tau_{V}$ units (the attenuation in the $V$ magnitude), by the following:

$$
\begin{aligned}
\frac{F_{\mathrm{H} \alpha}}{F_{\mathrm{H} \beta}} & =\frac{F_{\mathrm{H} \alpha, 0}}{F_{\mathrm{H} \beta, 0}} e^{-\left(\tau_{\mathrm{H} \alpha}-\tau_{\mathrm{H} \beta}\right)} \\
\tau_{\mathrm{H} \alpha} & =\tau_{V}\left(\frac{6563 \AA}{5500 \AA}\right)^{-0.7} \\
\tau_{\mathrm{H} \beta} & =\tau_{V}\left(\frac{4861 \AA}{5500 \AA}\right)^{-0.7},
\end{aligned}
$$

then

$$
\begin{aligned}
\tau & \equiv \ln \left(\frac{F_{\mathrm{H} \alpha}}{F_{\mathrm{H} \beta}} \frac{1}{2.88}\right) \\
& =\ln \left(\frac{F_{\mathrm{H} \alpha, 0}}{F_{\mathrm{H} \beta, 0}} \frac{1}{2.88}\right) e^{-\left(\tau_{\mathrm{H} \alpha}-\tau_{\mathrm{H} \beta}\right)} \\
& =\tau_{\mathrm{H} \beta}-\tau_{\mathrm{H} \alpha} \\
& =\tau_{V} / 4.84,
\end{aligned}
$$

and

$$
\begin{aligned}
\ln \left(\mathrm{SFR}_{1.4 \mathrm{GHz}} / \mathrm{SFR}_{\mathrm{UV}}\right) & =\tau_{V}(1500 \AA / 5500 \AA)^{-0.7} \\
& =2.48 \tau_{V} .
\end{aligned}
$$

Therefore, we define the quantities $\tau_{V \text {, line }}$ and $\tau_{V, \text { cont }}$ as

$$
\begin{aligned}
\tau_{V, \text { line }} & \equiv 4.84 \tau \\
\tau_{V, \text { cont }} & \equiv \ln \left(\mathrm{SFR}_{1.4 \mathrm{GHz}} / \mathrm{SFR}_{\mathrm{UV}}\right) / 2.48 .
\end{aligned}
$$

The two diagnostics correlate, although there is significant scatter. The supercompact UVLGs fall farther to the right side of the correlation defined by the other galaxies, toward higher continuum attenuation, and closer to the dashed line (where the line and continuum attenuation are equivalent). Supercompact UVLGs have nearly equal amounts of line and continuum attenuation, while other galaxies have higher line attenuation for a given continuum attenuation.

To compare with higher redshift LBGs: Reddy \& Steidel (2004) measure continuum attenuation as $\mathrm{SFR}_{\mathrm{X} \text {-ray }} / \mathrm{SFR}_{\mathrm{UV}} \sim$ 4.5-5, which is consistent with our sample's median $\mathrm{SFR}_{1.4 \mathrm{GHz}}$ / $\mathrm{SFR}_{\mathrm{UV}}=4.7$. In addition, Erb et al. (2003) find discrepancy between the UV and $\mathrm{H} \alpha$ SFR for LBGs at $z>2$, which they attribute to attenuation differences between regions giving rise to continuum versus line emission.

One explanation for why there is a mismatch between the line and continuum attenuation for the supercompact UVLGs might be the "birth cloud" description: ${ }^{14}$ for young systems, the

\footnotetext{
14 By "birth cloud," we refer to the scenario presented by Charlot \& Fall (2000). However, we relax their definition of birth cloud to include stars that may have been formed in earlier episodes, but enclosed within pockets of more recent star formation.
} 

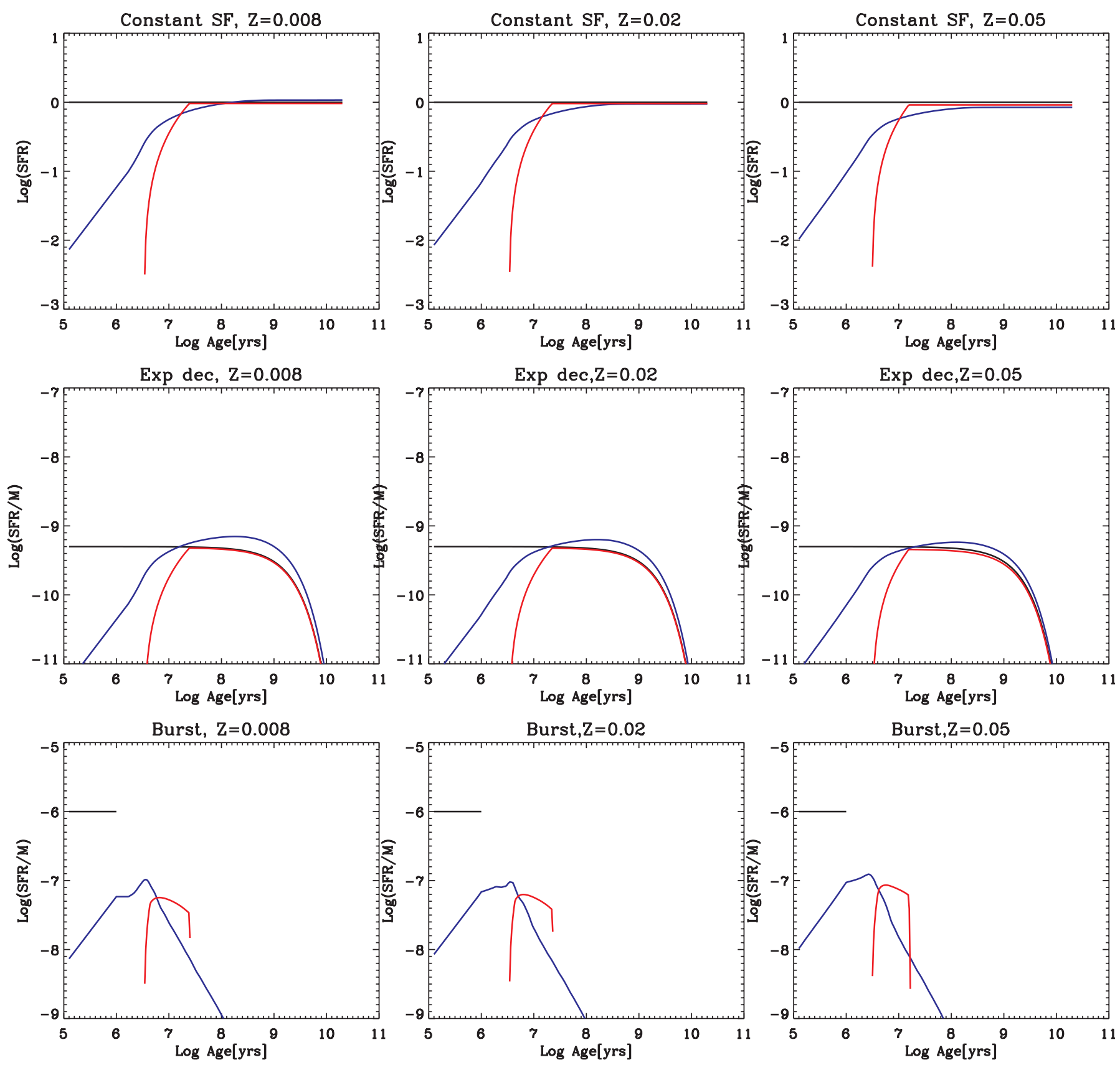

Lines: Model SFR, Radio SFR, UV SFR

FIG. 9.-Comparison of different star formation indicators in models: Radio SFR (red lines), UV SFR (blue lines), and model input (black lines). The rows display different star formation scenarios: constant, exponentially declining, and burst (top, middle, bottom), while the columns illustrate the effect of different metallicities: $Z=$ $0.008,0.02$, and 0.05 (left, middle, right).

continuum and line attenuation both arise from the same physical regions: in $\mathrm{H}$ II regions surrounding new stars and also the ISM; for older galaxies, the continuum is less attenuated, only by the ISM, while the emission lines are attenuated by both ISM and $\mathrm{H}$ II regions. If we assume that most of the stars in supercompact UVLGs are embedded in birth cloud regions, then we would expect the line and continuum attenuation measures to be similar. Since the continuum attenuation is lower than the line attenuation for other typical galaxies, the supercompact UVLGs would appear to have higher continuum attenuation than the typical galaxy.
Following this assertion a bit further, we introduce some models. We ran the Bruzual \& Charlot (2003) stellar population synthesis on three different star formation scenarios (an instantaneous burst, constant $1 M_{\odot} \mathrm{yr}^{-1}$, and exponentially declining with $\tau=$ $100 \mathrm{Myr})$, for different metallicities $(Z=0.008,0.02$, and 0.05 corresponding to $Z / Z_{\odot}=0.3,1$, and 2.5$)$ and different attenuation amounts, governed by

$$
\tau= \begin{cases}\tau_{V}(\lambda / 5500 \AA)^{-0.7} & \text { if } t \leq 10^{7} \mathrm{yr}, \\ \mu \tau_{V}(\lambda / 5500 \AA)^{-0.7} & \text { if } t>10^{7} \mathrm{yr},\end{cases}
$$




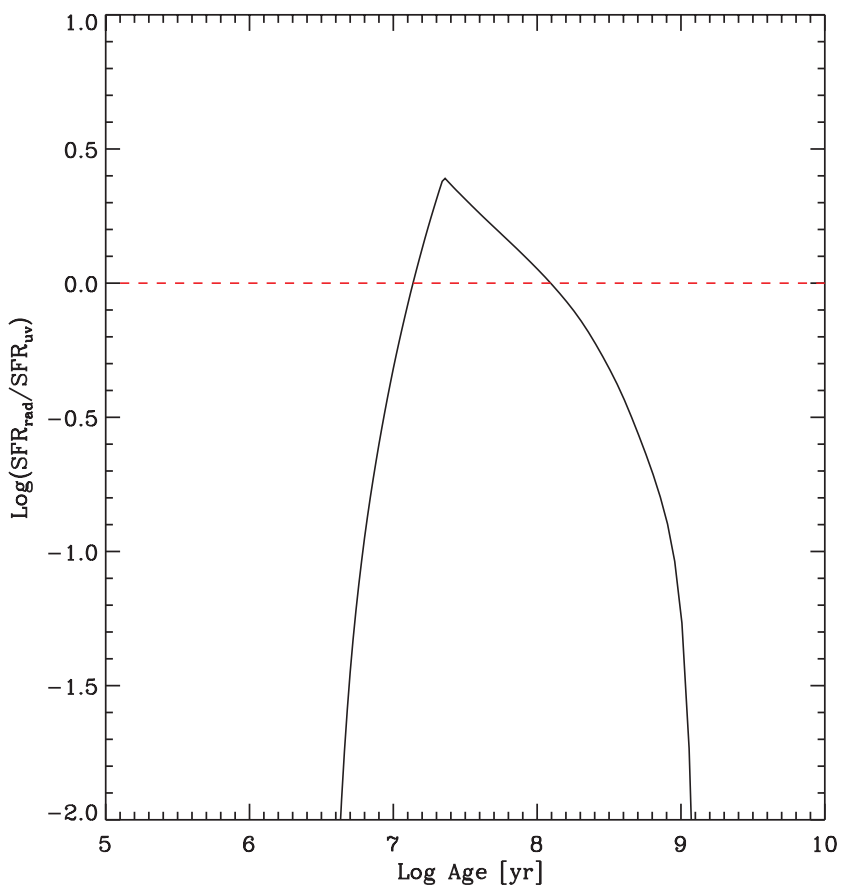

FIG. 10.- Radio to UV (unattenuated) SFR ratio vs. log(age) for the exponentially decaying star formation scenario with solar metallicity and decay constant of 100 Myr. The red dashed line marks where the radio SFR $=$ UV SFR. The plot demonstrates that for $t<10^{7} \mathrm{yr}$, the unattenuated UV SFR is greater than the radio SFR.

for a grid of values: $\tau_{V}=0,0.3,0.6,0.9,1.2,1.5$, and $\mu=$ $0,0.3,0.5,1.0$. The ISM contributes only a fraction, $\mu$, of the total attenuation. We use the Chabrier IMF, which produces results similar to the Kroupa IMF. From the UV luminosity, we calculate the SFR using equation (1). To simulate the radio emission we convert the supernova rate into a SFR using Condon (1992). However, we apply two additional conversion factors. First, the Condon (1992) formalism uses a Miller-Scalo (MS) IMF. We convert to the Chabrier (chab) IMF in the following way,

$$
\begin{aligned}
\operatorname{SFR}\left(M>5 M_{\odot}\right)_{\mathrm{chab}} & =\operatorname{SFR}\left(M>5 M_{\odot}\right)_{\mathrm{MS}} \frac{\int_{5 M_{\odot}}^{100 M_{\odot}} M^{-1.3} d M}{\int_{5 M_{\odot}}^{100 M_{\odot}} M^{-1.5} d M} \\
& =1.75 \mathrm{SFR}\left(M>M_{\odot}\right)_{\mathrm{MS} .}
\end{aligned}
$$

Second, to find the SFR that includes all, not just the high-mass $\left(M>5 M_{\odot}\right)$ stars, we correct by the factor

$$
\begin{aligned}
\operatorname{SFR} & =\operatorname{SFR}\left(M>5 M_{\odot}\right)_{\text {chab }} \frac{\int_{0.1 M_{\odot}}^{100 M_{\odot}} M \Psi(M) d M}{\int_{5 M_{\odot}}^{100} M_{\odot}} M^{-1.5} d M \\
& =2.44 \operatorname{SFR}\left(M>5 M_{\odot}\right)_{\text {chab }},
\end{aligned}
$$

where

$$
\Psi(\log M) \propto \begin{cases}e^{-\left(\log M-\log M_{c}\right)^{2} / 2 \sigma^{2}} & \text { if } M \leq 1 M_{\odot}, \\ M^{-1.3} & \text { if } M>1 M_{\odot}\end{cases}
$$

where $M_{c}=0.08 M_{\odot}$ and $\sigma=0.69$. In Figure 9, we show how well the derived UV and radio SFRs match the model SFRs (these are unattenuated SFRs). Since the conversions from luminosity or supernova rates to SFRs were derived for constant star formation at solar metallicity, we see that in the $Z=0.02$, constant star formation scenario, radio and UV SFRs match the model SFR best. Furthermore, the conversion from UV luminosity to SFR overestimates the UV SFR for $t \gtrsim 10^{7} \mathrm{yr}$ for the exponential decay scenario for all metallicity cases, demonstrating the limitations of determining SFRs from UV luminosities if the star formation history is unknown. We also show how the ratio of radio SFR to unattenuated UV SFR changes with time for the exponentially decaying star formation model (at solar metallicity) in Figure 10. For $t<10^{7} \mathrm{yr}$, the radio SFR is less than the unattenuated UV SFR.

Returning to the assertion that supercompact UVLGs may resemble the physical scenario where the majority of stars are within a birth cloud, we compare the continuum and line attenuations in Figure 11. The top shows the histograms of the ratio of continuum to line attenuation for the comparison samples, which peak around 0.4 (similar to the results by Calzetti et al. 1994). The supercompact UVLGs have a broader distribution, with a larger percentage of galaxies with higher continuum to line attenuation ratios. We overplot two theoretical curves for the line versus continuum attenuation on our data in Figure 11 (bottom). For our model data, the line attenuation is calculated from equation (6), where $\tau_{V}$ is varied for our model as described earlier. Similarly, for the continuum attenuation, the orange line marks the $t>10^{7} \mathrm{yr}$ case for $\mu=0.3$, and the blue line gives the $\leq 10^{7}, \mu=1.0$ case, both at $\lambda=1500 \AA$. We acknowledge that galaxies have far more complicated attenuations than described by these two curves. However, the location of the supercompact UVLGs on this plot agrees with the interpretation that most of the stars in these galaxies are still enclosed in birth clouds. Furthermore, Figure 11 displays the lower limit in continuum attenuation, because the radio SFR underestimates the total SFR for $t<10^{7} \mathrm{yr}$ (see Fig. 10).

Approaching the timescale issue of the supercompact UVLGs from a different angle, we introduce a few other star formation history indicators. $D_{n}(4000)$ measures the ratio of average flux densities $\left(F_{\nu}\right)$ between two narrow wavelength regions: $4000-$ 4100 and 3850-3950 ̊̊ (Balogh et al. 1999; Bruzual 1983). The latter region contains an accumulation of absorption features from ionized metals, creating a sudden discontinuity around $4000 \AA$. However, hotter stars can multiply ionize the metals, which reduces the opacity for this wavelength region and thereby eliminates the break. Young stellar populations lead to small $D_{n}(4000)$, while old, metal-rich galaxies have high $D_{n}(4000)$ values. The $\mathrm{H} \delta$ absorption strength $\left(\mathrm{H} \delta_{A}\right)$ also estimates the stellar population age. A-type stars have the most prominent Balmer absorption features. $\mathrm{O}$ and B stars dilute the absorption lines, since they dominate the spectrum yet have weak absorption. After the $\mathrm{O}$ and B stars perish $(\sim 0.1-1 \mathrm{Gyr}) \mathrm{H} \delta_{A}$ peaks; as the A stars finish their evolution the $\mathrm{H} \delta$ absorption strength decreases. The $\mathrm{H} \beta$ equivalent width $[\mathrm{EW}(\mathrm{H} \beta)]$ takes into account both the instantaneous SFR and the entire star formation history, and effectively measures the strength of the recent star formation activity compared to past. In Figure 12, we combine these different measures, plotting the $D_{n}(4000)$ versus $\mathrm{H} \delta_{A}$, and for the supercompact UVLGs the symbol size indicates $\mathrm{EW}(\mathrm{H} \beta)$. The dashed green line and solid magenta line follow an exponentially decaying (with a time constant of $2 \mathrm{Gyr}$ ) and instantaneous burst star formation history, respectively [they both increase in time from 

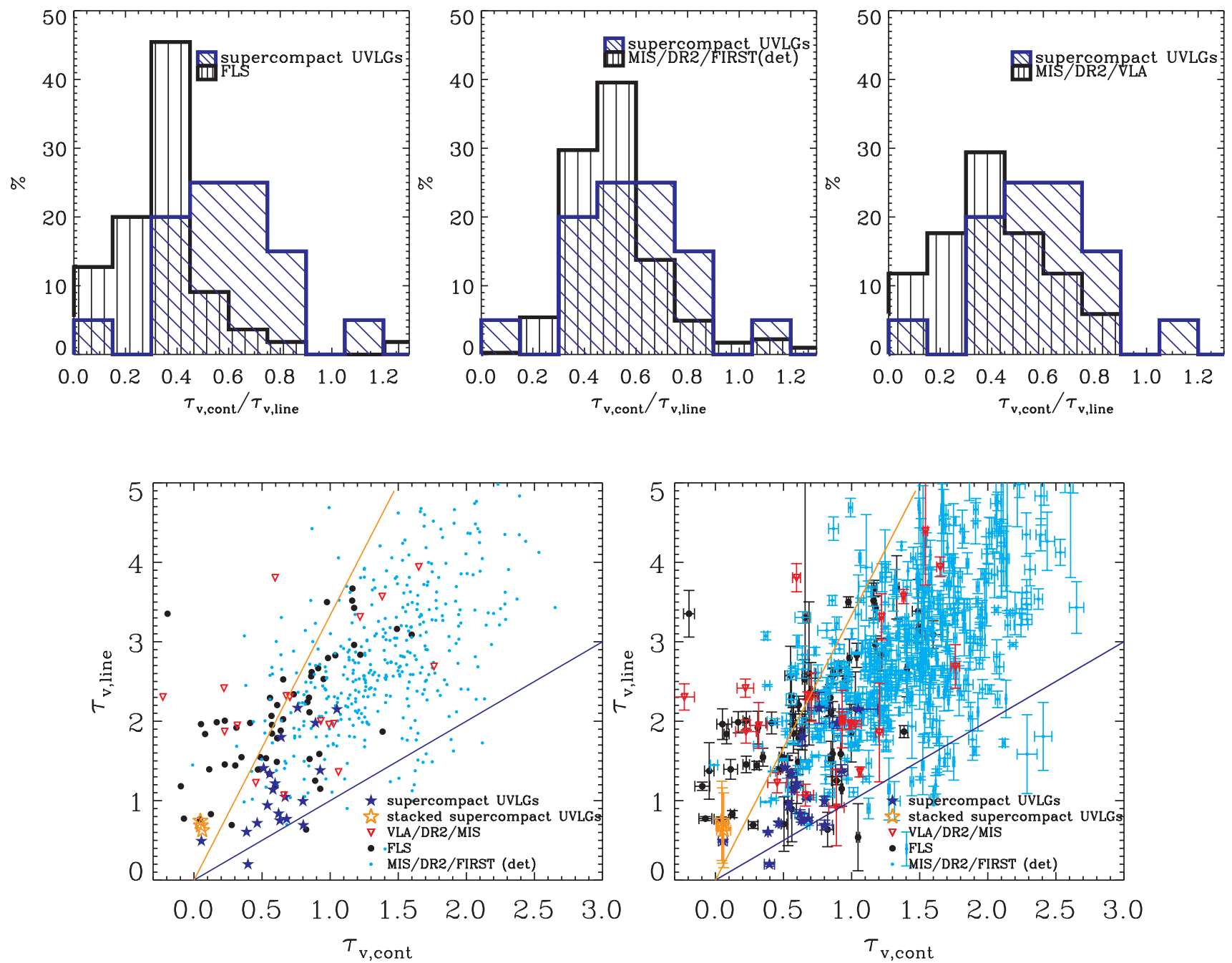

FIG. 11. - Comparison of two different measures of dust. $\tau_{V \text {, cont }} \equiv \ln \left(\mathrm{SFR}_{\mathrm{radio}} / \mathrm{SFR}_{\mathrm{uv}}\right) / 2.483$ measures attenuation of the continuum, while $\tau_{V, \text { line }} \equiv \ln [(\mathrm{H} \alpha /$ $\mathrm{H} \beta)(1 / 2.88)] 4.84$ measures attenuation in spectral lines. The histograms (top) show that the ratio of continuum to line attenuation peaks for normal galaxies (FLS, MIS/ DR2/FIRST[dets], and MIS/DR2/VLA, from left to right, respectively) 0.4, while the supercompact UVLGs have a broader distribution with a larger percentage of galaxies with higher ratios. Bottom: Orange line shows the theoretical curve for a galaxy where the attenuation in the continuum is $30 \%$ of the attenuation in the line ( $\mu=0.3$ ), while the blue line shows the case where the line and continuum are attenuated equally (all the stars are still in birth clouds, $\mu=1$ ). For a clearer view, the left panel excludes error bars and the MIS/DR2/FIRST stacked galaxies, while the right panel shows the full set with errors.

low $D_{n}(4000)$ to higher $D_{n}(4000)$ ]. Given their low $D_{n}(4000)$ and low $\mathrm{H} \delta_{A}$, the spectra of supercompact UVLGs are dominated by recent star formation.

Rosa-Gonzalez et al. (2007) study the radio properties of $\mathrm{H}$ II galaxies along with the $\mathrm{H} \beta$ equivalent width [ $\mathrm{EW}(\mathrm{H} \beta)$ ] and [O $\mathrm{III}] \lambda 5007$ to $\mathrm{H} \beta$ ratio. [O $\mathrm{OII}]$ is excited by massive stars, and strong $[\mathrm{O} \mathrm{III}]$ emission lines indicate early phases of star formation activity. While the $\mathrm{EW}(\mathrm{H} \beta)$ gauges present to past star formation, the $[\mathrm{O} \mathrm{III}] / \mathrm{H} \beta$ ratio detects recent starbursts. Therefore, single bursts would have high $\mathrm{EW}(\mathrm{H} \beta)$ and high $[\mathrm{O} \mathrm{III}] / \mathrm{H} \beta$, while bursts on underlying older stellar populations might have low $\mathrm{EW}(\mathrm{H} \beta)$ but high $\left[\mathrm{O}_{\mathrm{III}}\right] / \mathrm{H} \beta$. Figure 13 shows that supercompact UVLGs fall in the region with highest $\mathrm{EW}(\mathrm{H} \beta)$ and highest $[\mathrm{O}$ III $] / H \beta$; therefore, not only have they had recent bursts (as established already by their UV SFR), but their present to past SFRs exceed those observed in other star-forming galaxies. However, we note one important caveat: metallicity affects the $[\mathrm{O}$ III $] / \mathrm{H} \beta$ measure. The size of the symbols in Figure 13 indicates metallicity for the supercompact UVLGs. Lower metallicity results in higher gas temperatures, making it easier to collisionally excite the [O III] line. Given that supercompact UVLGs are metal-poor (Hoopes et al. 2006), we are less certain that the $[\mathrm{O}$ III $] / \mathrm{H} \beta$ indicates starbursts. Since the cause of the high $[\mathrm{O}$ III $] / \mathrm{H} \beta$ values is uncertain for the these galaxies we cannot confidently date the star formation, but compared to other galaxies supercompact UVLGs show evidence of recent activity.

$\mathrm{H}$ II galaxies have similar $[\mathrm{O}$ III] $/ \mathrm{H} \beta$ properties ( Rosa-Gonzalez et al. 2007); however, these galaxies have FUV surface brightnesses (or SFRs per unit area) an order of magnitude lower than supercompact UVLGs and FUV luminosities 2 orders of magnitude lower than the supercompact UVLGs (Hoopes et al. 2006). We briefly consider the connection between supercompact UVLGs and $\mathrm{H}$ II galaxies. The spectra of $\mathrm{H}$ II galaxies resemble $\mathrm{H}$ II regions, where embedded young stars dominate the emission. The radio emission in these galaxies is dominated by the thermal, rather than synchrotron, contribution. Since the thermal bremsstrahlung spectrum is characterized by a $\nu^{-0.1}$ power law, while synchrotron emission follows $\nu^{-0.8}$, the spectral index $(\alpha$, where $S_{\nu} \propto \nu^{\alpha}$ ) can discern which mechanism is responsible for the radio emission. Furthermore, an accurate measure of the SFR from 

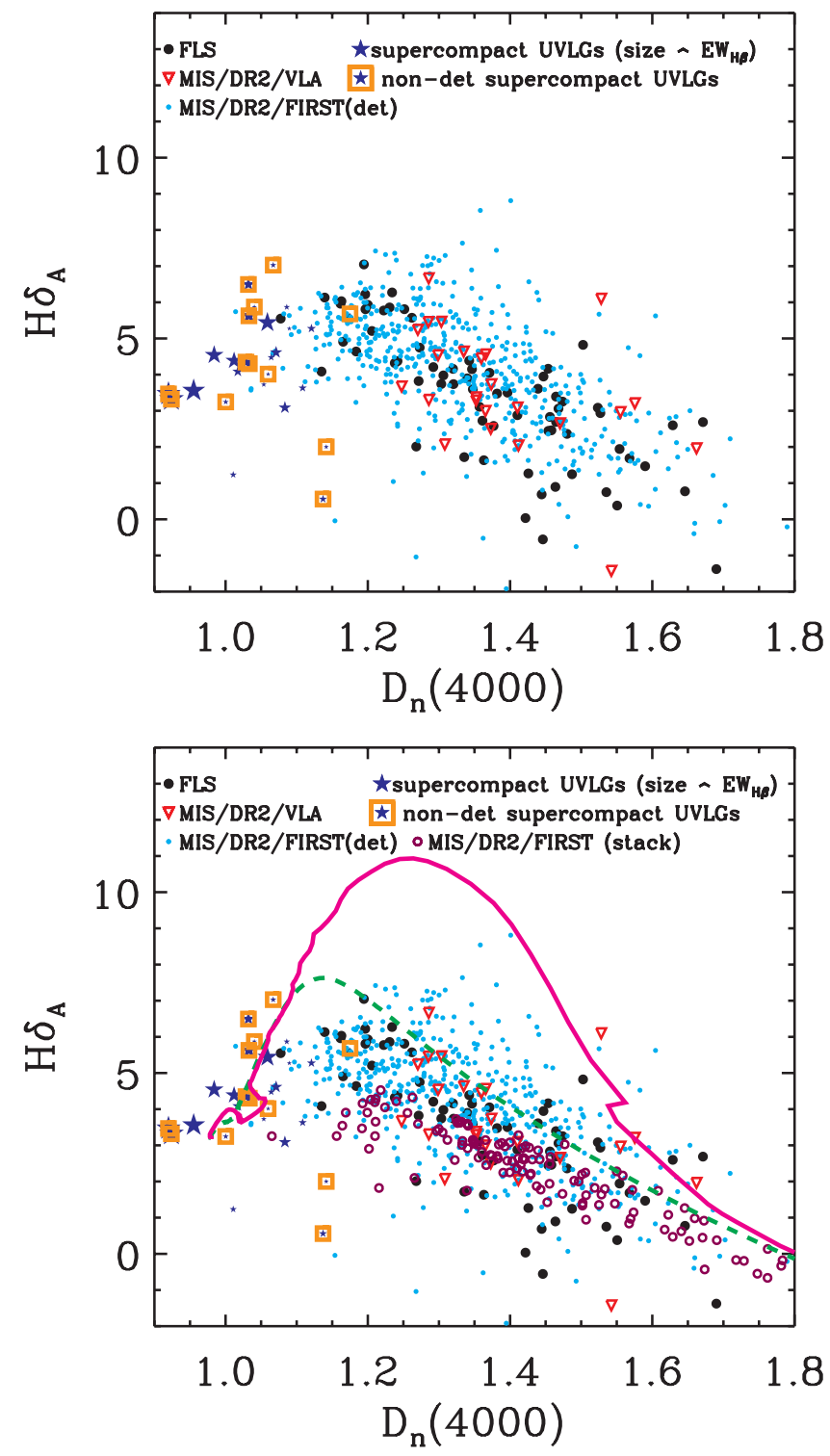

FIG. 12.-The $4000 \AA$ A break index vs. the Balmer absorption line strength. The typical galaxies (black dots and inverted red triangles, as described in Fig. 5) have decreasing $\mathrm{H} \delta_{A}$, indicating the expiration of stars with strong Balmer absorption, with increasing $D_{n}(4000)$. Supercompact UVLGs (blue stars) may have undergone a very recent burst. The sizes of the stars reflect the $\mathrm{H} \beta$ equivalent width, another indicator of recent to past star formation. In the bottom plot, we include the stacked MIS/DR2/FIRST galaxies and the models. The magenta solid line refers to an instantaneous burst of star formation with solar metallicity, whereas the green dotted line demonstrates exponentially decaying star formation with a decay constant of 2 Gyr and solar metallicity.

the radio data depends on understanding the contribution of thermal versus nonthermal; current conversions assume that $90 \%$ of the radio is nonthermal at $20 \mathrm{~cm}$. We have acquired $6 \mathrm{~cm}$ radio data, in order to determine spectral indices for the supercompact UVLGs, and our preliminary results indicate that the nonthermal contribution dominates the spectrum, consistent with the normal $\mathrm{FIR} /$ radio flux ratios that we measure.

We have outlined two explanations for why the radio luminosities (for given UV luminosities) might be low for the supercompact UVLGs: less dust attenuation and recent starburst activity. If we assume that the radio-IR correlation implies consistent SFRs, then the issue of recent star formation is less relevant.

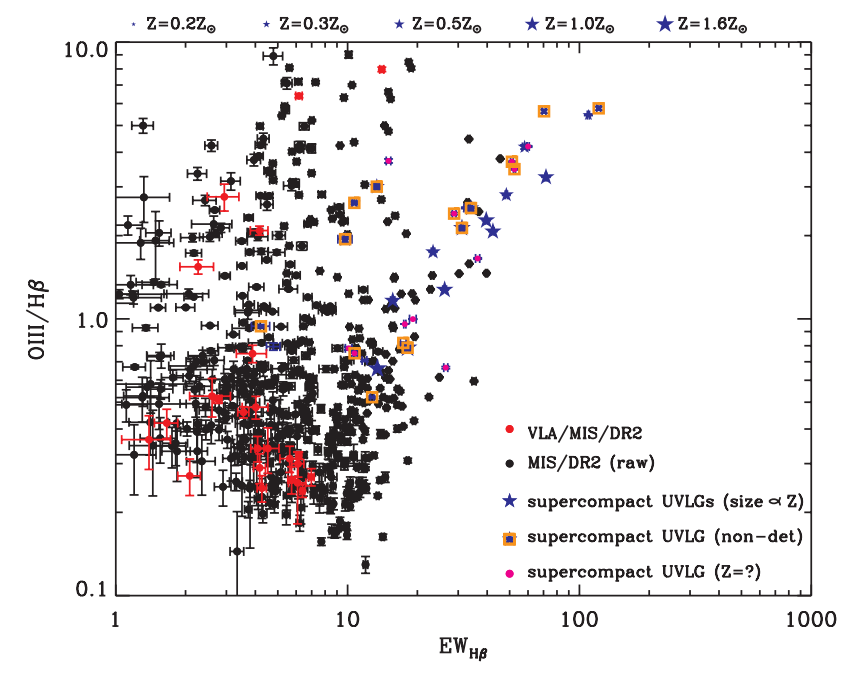

FIG. 13.- $\mathrm{O}$ III/ $/ \mathrm{H} \beta$ vs. $\mathrm{EW}(\mathrm{H} \beta)$ can segregate star formation histories. Bursts, on underlying older stellar populations have high $\mathrm{O}$ III $/ \mathrm{H} \beta$, but low $\mathrm{EW}(\mathrm{H} \beta)$ while single, recent bursts have high $\mathrm{O}$ III $/ \mathrm{H} \beta$ and $\mathrm{EW}(\mathrm{H} \beta)$. The supercompact UVLGs (blue stars) seem to be the latter, although low metallicity may contribute to high $\mathrm{O}$ III $/ \mathrm{H} \beta$ values more than burst history. To qualitatively test this point, we indicate metallicity by symbol size for the supercompact UVLGs (the magenta points have undetermined metallicities) and a rough guide relating the symbol size to metallicity is shown at the top of the panel $\left(Z_{\odot}=8.69\right)$. The black dots are unstacked MIS/DR2/FIRST galaxies.

Although we will investigate this further in a future paper, our IR data suggests low dust attenuation is the more plausible explanation for our observations.

\section{CONCLUSIONS}

Our study has combined the $1.4 \mathrm{GHz}$ radio data with UV, several spectral measures, and IR (in some cases) to understand the dust attenuation properties and star formation histories of supercompact UVLGs, compared to typical, local galaxies. For galaxies with no dust attenuation, the UV SFR should measure the total SFR; for galaxies with stellar populations older than $\sim 10^{7}-$ $10^{7.5} \mathrm{yr}$, the $1.4 \mathrm{GHz}$ SFR should measure the total SFR (see Figs. 9 and 10). In light of these assumptions, we note the following results:

1. The supercompact UVLGs are consistent with radio-IR relationship found by Yun et al. (2001). However, to compare the IR SFR with radio SFR, we need to complete further analysis of the IR data. This result suggests that the radio continuum can be used as a proxy for the FIR and can (in conjunction with the FUV) be used to determine the SFR and FUV extinction.

2. We derive that the average $L_{\mathrm{rad}}=5.8 \times 10^{22} \mathrm{~W} \mathrm{~Hz}^{-1}$, which is comparable to the value found by Reddy \& Steidel (2004): $5.9 \times 10^{22} \mathrm{~W} \mathrm{~Hz}^{-1}$ for stacked $1.5 \leq z \leq 3.0$ LBGs. The implied SFRs are in the range of $10-100 M_{\odot} \mathrm{yr}^{-1}$, similar to LBGs.

3. For a given FUV luminosity, the radio luminosity appears to be lower than what is expected using relations derived by Wang \& Heckman (1996) and Martin et al. (2005). While other samples of galaxies have points scattered around the relation, the supercompact UVLGs have $L_{\mathrm{rad}}$ that fall below the relation (see Fig. 7).

4. Our average $\mathrm{SFR}_{\mathrm{rad}} / \mathrm{SFR}_{\mathrm{UV}} \sim 4.7$, consistent with LBGs (Reddy \& Steidel 2004). Compared to typical local galaxies with such high SFRs (i.e., LIRGs), the supercompact UVLGs have 
significantly less FUV attenuation. The combination of modest FUV attenuation with high SFR is similar to what is observed in LBGs.

5. Supercompact UVLGs have lower line attenuation, but comparable continuum attenuation to other typical galaxies (Fig. 8). We compute the line attenuations from Balmer decrements $(\tau=$ $\ln [(\mathrm{H} \alpha / \mathrm{H} \beta)(1 / 2.88)])$. The $\mathrm{SFR}_{\mathrm{rad}} / \mathrm{SFR}_{\mathrm{UV}}$ quantity estimates the continuum attenuation. We consider the effect of birth clouds on line versus continuum attenuation to derive simple theoretical curves, which we overplot with the data in Figure 11. The location of the supercompact UVLGs on the $\tau_{V \text { line }}-\tau_{V, \text { cont }}$ plot is consistent with the interpretation that stars in supercompact UVLGs are embedded in birth clouds.

6. Compared to our set of comparison galaxies, defined in $\S \S 2.2-2.4$, supercompact UVLGs have lower $D_{n}(4000)$ and lower $\mathrm{H} \delta_{A}$ (implying the presence of young $\mathrm{O}$ and $\mathrm{B}$ stars), higher $\mathrm{EW}(\mathrm{H} \beta$ ), and higher $[\mathrm{O} \mathrm{III}] / \mathrm{H} \beta$ (suggesting that most of the stars were formed recently).
These results consistently indicate that supercompact UVLGs are low-dust systems with recent star formation, and reinforce their connection with high-redshift LBGs.

GALEX (Galaxy Evolution Explorer) is a NASA small explorer, launched in 2003 April. We gratefully acknowledge NASA's support for construction, operation, and science analysis for the GALEX mission, developed in cooperation with the Centre National d'Etudes Spatiales of France and the Korean Ministry of Science and Technology. We thank Michael Blanton for access to the IDL kcorrect (ver. 4.1.4) analysis package. We recognize that funding for the SDSS archive has been provided by the Alfred P. Sloan Foundation, the Participating Institutions, the National Aeronautics and Space Administration, the National Science Foundation, the US Department of Energy, the Japanese Monbukagakusho, and the Max Planck Society.
Adelberger, K. L., Steidel, C. C., Pettini, M., Shapley, A. E., Reddy, N. A., \& Erb, D. K. 2005, ApJ, 619, 697

Balogh, M. L., et al. 1999, ApJ, 527, 54

Becker, R. H., White, R. L., \& Helfand, D. J. 1995, ApJ, 450, 559

Bell, E. F. 2003, ApJ, 586, 794

Best, P. N., Kauffmann, G., Heckman, T. M., \& Ivezić, Ž. 2005, MNRAS, 362, 9

Blanton, M. R., et al. 2003, AJ, 125, 2348

Brinchmann, J., et al. 2004, MNRAS, 351, 1151

Bruzual, G. 1983, ApJ, 273, 105

Bruzual, G., \& Charlot, S. 2003, MNRAS, 344, 1000

Calzetti, D., Kinney, A. L., \& Storchi-Bergmann, T. 1994, ApJ, 429, 582

Charlot, S., \& Fall, S. M. 2000, ApJ, 539, 718

Condon, J. J. 1992, ARA\&A, 30, 575

Condon, J. J., et al. 2003, AJ, 125, 2411

Dijkstra, M., Haiman, Z., \& Spaans, M. 2006, ApJ, 649, 37

Efron, B. 1982, The Jackknife, the Bootstrap, and Other Resampling Plans (Philadelphia: SIAM)

Erb, D. K., Shapley, A. E., Steidel, C. C., Pettini, M., Adelberger, K. L., Hunt, M. P., Moorwood, A. F. M., \& Cuby, J.-G. 2003, ApJ, 591, 101

Furlanetto, S. R., Schaye, J., Springel, V., \& Hernquist, L. 2005, ApJ, 622, 7

Garland, C. A., Pisano, D. J., Williams, J. P., Guzmán, R., \& Castander, F. J. 2004, ApJ, 615, 689

Garland, C. A., Williams, J. P., Pisano, D. J., Guzmán, R., Castander, F. J., \& Brinkmann, J. 2005, ApJ, 624, 714

Gil de Paz, A., \& Madore, B. F. 2005, ApJS, 156, 345

Guzmán, R. 2005, in Proc. ESO Workshop, Multiwavelength Mapping of Galaxy Formation and Evolution, ed. R. Bender \& A. Renzini (Berlin: Springer), 191

Guzmán, R., Östlin, G., Kunth, D., Bershady, M. A., Koo, D. C., \& Pahre, M. A. 2003, ApJ, 586, L45

Heckman, T. M., et al. 2005, ApJ, 619, L35

Hoopes, C. G., et al. 2006, ApJS, 173, 441

Hopkins, A. M., et al. 2003, ApJ, 599, 971
Kennicutt, R. C. 1998, ARA\&A, 36, 189

Kroupa, P. 2001, MNRAS, 322, 231

Lowenthal, J. D., et al. 2005, in ASSL 329, Starbursts: From 30 Doradus to Lyman Break Galaxies, ed. R. de Grijs \& R. M. González Delgado (Dordrecht: Springer), 17

Martin, D. C., et al. 2005, ApJ, 619, L59

Mori, M., \& Umemura, M. 2006, CRAL-2006: Chemodynamics, from First Stars to Local Galaxies, ed. E. Emsellem et al. (Les Ulis: EDP Sciences), 221 Morrissey, P. 2007, ApJS, 173, 682

Perez-Gonzalez, P. G., et al. 2003, MNRAS, 338, 525

Phillips, A. C., Guzman, R., Gallego, J., Koo, D. C., Lowenthal, J. D., Vogt, N. P., Faber, S. M., \& Illingworth, G. D. 1997, ApJ, 489, 543

Reddy, N. A., \& Steidel, C. C. 2004, ApJ, 603, L13

Rosa-Gonzalez, D., Schmitt, H. R., Terlevich, E., \& Terlevich, R. 2007, ApJ, 654,226

Rosenberg, J. L., Ashby, M. L. N., Salzer, J. J., \& Huang, J.-S. 2006, ApJ, 636, 742

Salpeter, E. E. 1955, ApJ, 121, 161

Schmitt, H. R., et al. 2006, ApJ, 643, 173

Smail, I., et al. 2007, ApJ, 654, L33

Steidel, C. C., Adelberger, K. L., Giavalisco, M., Dickinson, M., \& Pettini, M. 1999, ApJ, 519, 1

Steidel, C. C., Adelberger, K. L., Shapley, A. E., Pettini, M., Dickinson, M., \& Giavalisco, M. 2000, ApJ, 532, 170

Steidel, C. C., Pettini, M., \& Hamilton, D. 1995, AJ, 110, 2519

Sullivan, M., et al. 2001, ApJ, 558, 72

Tremonti, C. A., et al. 2004, ApJ, 613, 898

Wang, B., \& Heckman, T. M. 1996, ApJ, 457, 645

White, R. L., Helfand, D. J., Becker, R. H., Glikman, E., \& deVries, W. 2006, ApJ, 654, 99

Wyder, T. 2007, ApJS, 173, 293

Yun, M. S., Reddy, N. A., \& Condon, J. J. 2001, ApJ, 554, 803 